\title{
Mapping our underlying cognitions and emotions about good environmental behaviour. Why we fail to act despite the best of intentions.
}

\author{
Nicola Power, Geoff Beattie and Laura McGuire \\ Department of Psychology \\ Edge Hill University
}

\begin{abstract}
Despite the widespread recognition of climate change as the single biggest global threat, the willingness of people to change their behaviour to mitigate its effects is limited. Past research, often focussing on specific categories of behaviour, has highlighted a very significant gap between people's intentions to behave more sustainably and their actual behaviour. This paper presents a new approach to this issue, by using more open-ended questions to map a much broader range of cognitions and emotions about good environmental behaviour. Two key findings emerged. Firstly, participants were aware of the contradiction between their level of concern about the environment and their willingness to act in more sustainable ways. The qualitative analysis further revealed that this discrepancy often hinged on a lack of knowledge about how to act more sustainably; the analysis also revealed a desire for more information about genuinely green behaviour. Secondly, pro-environmental behaviour was often conceptualised by participants in essentially 'social' terms; anticipated emotions relating to sustainable/non-sustainable behaviour were as closely tied to the behaviour of one's peers as to one's own behaviour. This finding suggests that we must highlight the social dimension in any interventions to increase sustainable behaviours amongst the public.
\end{abstract}

\section{Introduction}

Climate change is the single biggest threat that we, as a species, have faced. The scientific evidence for climate change, its causes and its potential consequences, is now unambiguous (indeed, as unambiguous as science can ever be) and the evidence around climate change has been carefully tested and marshalled by the Intergovernmental Panel on Climate Change (IPCC) in a succession of reports. Given how scientifically compelling the case is, there really should be no remaining doubt about either the seriousness or the immediacy of this problem or about the necessity for large-scale behavioural adaptation on the public's part to 
reduce greenhouse gases. The IPCC say that they are 'now 95 percent certain that humans are the main cause of current global warming.' (IPCC 2015: v; italics added). They do, however, go on to say that, "we have the means to limit climate change and its risks, with many solutions that allow for continued economic and human development.' (IPCC 2015: v). It is clear that the means to limit climate change must be a number one global priority, and that engaging the public in this so that it leads to large-scale behaviour change is of the utmost importance. However, one feature of the myriad climate change problem that perhaps stands out more than any other is the fact that although the role of human activity in its causation is both "clear and growing' (IPCC 2015: v), evidence for large scale behavioural change is absent (see Beattie 2010) .

The IPCC outline a range of aspects of human activity that contribute to greenhouse gas emissions (GHG), and thereby affect climate change. These cover broad demographic and economic variables like population size, economic activity and energy use, as well as land use patterns, technology and climate change policy. However, they also identify another major factor - 'lifestyle', where 'lifestyle' reflects aspects of the behavioural choices that people make in their everyday lives that have an effect on GHG. Of course, 'lifestyle' could well be a critical variable in this context because it could potentially change (and change faster and more precipitously than many of the other factors like 'population size' or 'land use patterns'). Indeed, the IPCC identified 'lifestyle' as one of the common enabling factors that underpin adaptation and mitigation responses. However, clearly, we need a much better understanding of the variables that influence lifestyle choices, and particularly those choices with a direct bearing on GHG, if we are to prevent further changes in our climate. What we do now know is that 'lifestyle', as a form of individual adaptation has not changed in the ways that one might have predicted given the immediacy and the seriousness of the threat (Beattie, 2010). This is despite all of the impressive scientific evidence, the various government and commercial campaigns (e.g. the Unilever Sustainable Living Plan 2013), and the vast amounts of publicity some involving highly emotive and dramatic film content, including the international awardwinning documentary 'An Inconvenient Truth'. This Academy Award winning film clearly has an immediate psychological impact on emotion, and feelings of social responsibility, but not necessarily on behavioural choices, consumer habits or long-term behavioural adaptation (see Beattie 2011; Beattie, Sale and McGuire 2011). 
The IPCC (2015) assert that we can limit the effects of climate change by altering our behaviour and taking action now. This can be achieved by changing our collective behaviour in the direction of more sustainable, everyday goals, for example, by using public transport rather than private vehicles (Wall, Devine-Write and Mill 2007), by increasing recycling (Elgaaied 2012) or by changing consumer choices to approach a more carbon neutral purchasing pattern (Walker and King 2008). There are many things that we could do, and indeed, there has been significant local change in some countries, but the change is not of the order that is required to ameliorate the further deleterious effects of climate change. One example, perhaps, illustrates the problem. One of the leading multinationals, Unilever, in their Sustainable Living Plan set out to halve the greenhouse gas impact of their products across the lifecycle by 2020 (2013: 16) In pursuit of this goal, they reduced greenhouse gas emissions from their manufacturing chain and deforestation. They opted for more environmentally friendly sourcing of raw materials, doubled their use of renewable energy, and produced concentrated liquids and powders. They reduced greenhouse gas emissions from transport and refrigeration. They also restricted employee travel. The result of all of these initiatives was that their 'greenhouse gas footprint impact per consumer....increased by around 5\% since 2010' (2013: 16). They conclude pessimistically 'We have made good progress in those areas under our control but...the big challenges are those areas not under our direct control like.....consumer behaviour' (2013:16).

It seems that consumers are either not getting the message, or if they are, they are not acting on it. Despite the widespread recognition of climate change as a global problem, there remains to be a persistent psychological problem that scholars have struggled to resolve: why do expressed positive attitudes to sustainability and wilful intentions to act in a sustainable manner not always lead to increased sustainable behaviour (Bamberg 2003)? Ethically minded consumers intend to buy low carbon products but fail to take these products to the till (Auger and Devinney 2007). We seem to recognise a need to make more sustainable life choices, but fail to put these intentions into practice. The gap between attitudes, intentions and behaviour is an enduring quandary in the psychological literature. What is different in the case of sustainability is that we do not have the luxury of deliberation on this question. Action on climate change is required urgently and so the attitude/intention-behaviour gap needs to be closed.

There have been a number of attempts at an explanation. One approach is to consider whether we are actually measuring underlying attitudes in the right way. Some have suggested 
that we need to measure 'implicit attitudes', as well as self-reported 'explicit' attitudes. Implicit attitudes are those that seem to be unconsciously rather than consciously held, and thus not readily reported. The classic definition of 'attitude' in psychology comes from Gordon Allport (1935). He defined it as 'a mental and neural state of readiness organized through experience, exerting a directive or dynamic influence upon the individual's response to all objects and situations with which it is related' (1935: 810). It has been pointed out that there is nothing in Allport's definition that formally excludes a possible unconscious component (Beattie 2012a: 124). After all, why should we be consciously aware of our 'neural state of readiness', and in a position to report it in attitude questionnaires whenever and wherever required? Implicit attitudes have been measured using the Implicit Association Test (Greenwald, McGhee and Schwartz 1998). This is designed to probe the associative connections between concepts (for example high/low carbon products and the evaluative concepts of $\mathrm{good} / \mathrm{bad}$ ) and it has been found repeatedly that in the domain of sustainability explicit (self-report) attitudes and implicit attitudes (measures using the IAT) do not correlate (Beattie 2010; Beattie and Sale 2009, 2011; Beattie and McGuire 2015). Further, implicit attitudes seems to affect visual attention to climate change images in a way that self-report attitudes do not (Beattie and McGuire 2012), as well as visual attention to carbon footprint information on products, at least in the short time frames important in actual shopping (Beattie, McGuire and Sale 2011; Beattie and McGuire 2015). They also seem to predict behavioural choice when there is a degree of time pressure in that choice (Beattie and Sale 2011). In a different domain - the selection of candidates for academic posts, implicit racial attitudes, rather than explicit racial attitudes, seems to predict a racial bias in the selection of candidates for shortlisting (Beattie 2012a; Beattie, Cohen and McGuire 2013). This implicit racial bias could well predict the under-representation of individuals from certain racial or ethnic backgrounds in particular occupations.

There is thus evidence that implicit attitudes might have some role to play in influencing actual behaviour in a number of domains. However, that is not to say that explicit attitudes do not themselves have a very significant role to play in this regard. Greenwald et al (2009) conducted a meta-analysis on the predictive validity of the IAT and explicit measures of attitudes, and concluded that, in general, when the IAT and explicit attitude measures are combined they are better predictors of behaviour than either measure alone. Fazio (1990) has argued that explicit measures are much better predictors of behaviour when people have the motivation and opportunity to deliberate. Clearly, some behavioural choices linked to 
sustainability do require extensive deliberation (and require high levels of motivation as well as the opportunity for such deliberation) before a behavioural choice is made. The choice of a new car, the installation of solar panelling, or the decision to use public transport all clearly require a degree of deliberation, so explicit attitudes might well be very significant in these sorts of contexts (although implicit attitudes might well still be significant, see Barthes 1957; Hegarty 2011, for a discussion of the role of implicit values in the purchase of a brand of car). However, if we are to consider explicit attitudes to pro-environmental behaviour and sustainability are we confident that we have genuinely mapped out the full conceptual domain that we need to consider? It could be argued that previous research addressing the intentionbehaviour gap has done so through the experimental manipulation of a relatively small set of known variables. Perhaps, the time has come to adopt a more exploratory approach to this issue, to identify how individuals conceptualise pro-environmental behaviour more broadly. For this reason, this study uses an open-question online survey format to generate free recall responses from participants to explore their awareness and understanding of what proenvironmental behaviour actually is. It explores the anticipated consequences for our participants of adopting, or not adopting, such behaviour, as well as the anticipated emotions that may arise should they adopt or fail to adopt pro-environmental behaviour, as well as their overall general conceptualisation of global warming and pro-environmental behaviour. This differs from previous research, by offering a more 'neutral' exploration of the intentionbehaviour gap, rather than creating experimental manipulations that may prompt participants to express how they ought to think and behave.

\section{The intention-behaviour gap and sustainable decision-making}

It is critical to understand the relationship between intentions and behaviour when trying to target interventions to facilitate pro-environmental (PE) behaviour change. Some have argued that more sustainable behaviour can be achieved through the provision of full information on facts relating to climate change (Peattie 2001). This could be through informative documentaries and advertisement campaigns that clearly state the facts of global warming. This 'rational' approach to encourage sustainable choices is based upon the assumption that one's intentions to act in a certain way, which are based on cognitive evaluations of the situation, are proxy to behaviours (Ajzen 1991). People make rational choices and so increased knowledge about climate change should activate intentions and behaviour to act in a more sustainable way. However, in numerous domains it has been found 
that intentions and behaviour are not correlated (Bamberg, 2003). This suggests that cognitive evaluations are not the only factor influencing PE choices.

Some have suggested that intentions to act are irrelevant if those making decisions fail to hold 'implementation intentions' that refer to knowledge on how to act in a sustainable manner (Carrington, Neville and Whitwell 2010). A lack of understanding on PE behavioural options can derail the intention to act in a sustainable way, as individuals simply do not know what to do. It is also clear that PE behaviour can be influenced by a number of factors that go beyond explicit, cognitive evaluation, including such things as emotions (Koenig-Lewis, Palmer, Dermody and Ubye 2014) and perceived time for taking effective action (Polonsky, Vocino, Grimmer and Miles 2014). As the issue of climate change is a real, complex problem it means that there are a myriad of variables (some cognitive, others emotional, some highly personal, others culturally specific) that may shape one's attitudes, intentions and associated behaviour (Carrington, et al. 2010). As such, research that seeks to investigate the relationship between attitudes, intentions and behaviours by simply exploring participants' views about specific, isolated behaviours, (for example, recycling), may well suffer by this reductionism, by essentially excluding all of these other more general factors that can play a role in broad conceptualizations of the problem. This paper, therefore, explores rather than tests participants' attitudes, emotions and reported behaviours, in an attempt to approximate a more 'real-world' conceptualisation of PE behaviour. The results of this study could potentially help us to understand where to target interventions that may help to close the gap between PE intentions and behaviour. Firstly, however, this paper will discuss some of the previous research that has initially sought to test what influences sustainable choices.

\section{The Theory of Planned Behaviour (TPB)}

The Theory of Planned Behaviour (TPB) (Ajzen 1991) is one of the most influential models that has been used to attempt to explain and predict variations in sustainable behaviour. It is a 'rational' model of decision making that describes how one's intention to perform a given behaviour is influenced by three factors: (i) attitude towards the behaviour, (ii) subjective (social) norms; and (iii) perceived behavioural control (Ajzen 1991). If a behaviour is evaluated positively (attitude), if it is perceived as socially acceptable (subjective norms) and if the individual believes they have the capacity to execute the behaviour (behavioural control), then people will hold high intentions to perform the relevant behaviour. Mancha and Yoda (2015) used the TPB to explain cultural variations in PE behaviour. They found that of the 
three factors that influence intentions, one's attitude towards sustainable choices was more closely tied to intentions in individualist (e.g. Western) cultures, whereas perceived social norms relating to $\mathrm{PE}$ behaviour were more closely linked to intentions in collectivist cultures (e.g. India). Research using the ТPB could be useful for targeting interventions by aligning interventions to increase PE choice with culturally relevant markers. The focus could be on targeting attitude change in individualist cultures, for example, via the use of persuasive messages, and focussing on social interventions to increase perceived social norms relating to sustainable behaviour in more collectivist cultures.

A number of studies using the TPB to investigate PE behaviour have extended the model to include additional variables. Donald, Cooper and Conchie (2014) found that habit (i.e. past behaviour) could be added to the TPB to improve predictions on the likelihood of private car use over public transport to commute to work. It has also been found that one's affective connection to the environment (i.e. how closely one affiliates their personal identity with the environment) can increase explained variance in behaviour using the TPB (Hinds and Sparks 2008). Other variables that have been added to the TPB for sustainable choice include moral norms (Cheng and Tung 2010), environmental concern (Fransson, Davidsson, Marell and Garling 1994), environmental self-identity (Hinds and Sparks 2008) and descriptive norms with regards to others' behaviour (Donald et al. 2014). The decision to adopt PE behaviour is clearly a complex choice with many interacting variables, which are often difficult to model in a laboratory setting.

Although the TPB has made some progress in explaining how variations in attitudes, social norms and behavioural control may influence PE behaviour and intentions, there are a number of limitations with this model. Firstly, it is based on an assumption that PE intentions will lead to PE behaviours. However, as already mentioned, there is generally a poor relationship between environmental intent and behaviour (Bamberg 2003) and so this assumption could be said to be inherently flawed. Secondly, the capacity for the model to explain variations in behaviour is relatively limited. A meta-analysis of studies using the TPB concluded that it only explained around $40 \%$ of variance in behavioural intent and $29 \%$ of the variance in actual behaviour (Armitage and Conner 2001). This suggests that there are a number of variables that the TPB does not measure that influence behaviour. A third limitation is that the TPB is a highly rational model of decision making that ignores variables relating to emotional processing, which have been found to play a key role in decision making relating to environmental choices (Richard, van der Pligt and De Vries 1996). 


\section{Extending the TPB: The Model of Goal-Directed Behaviour}

In an attempt to address some of the problems outlined with the TPB, Perugini and Bagozzi (2001) created the Model of Goal-Directed Behaviour (MGB), which extends the TPB to include: (i) desire; (ii) anticipated negative emotions; (iii) anticipated positive emotions; and (iv) the frequency and recency of similar past behaviour (Prugini and Bagozzi 2001). Desire is distinct from 'intention' and relates to goal aspirations (Perugini and Bagozzi 2004), and is defined as "the motivational state of mind, wherein appraisals and reasons to act are transformed into motivation to do so" (Perugini and Bagozzi 2001: 84). It is a motivational state that aligns one's cognitive goals and emotions (Elliot 2006), which supports the notion that 'intentions' on their own do not necessarily lead to behaviour. Individuals must also have motivated desires to achieve the behavioural goal. When individuals are asked to think about desirable behaviours they tend to think about abstract and future-oriented goals that one hopes to achieve (Lutchyn and Yzer 2011), for example to 'lose weight'. This compares to intended behaviours, which are concrete and anchored to plans and specific behaviours (Perugini and Bagozzi 2001); for example to go the gym three times per week. Desires, compared to intentions, are: (i) less performable as one can desire something without knowing how to obtain it; (ii) more abstract as they are less connected to specific actions; and (iii) often future oriented or time indefinite (Perugini and Bagozzi 2004). For example, you and your friend may share the desire to be more 'environmentally friendly', which is a superordinate, abstract goal with no specific temporal framing; however our intentions to achieve this goal may be very different. You may intend to increase the amount that you recycle each week whereas your friend may intend to take the bus to work once a week rather than use her car, both performable, concrete goals set in the present/near-future. Research utilising the MGC has supported this distinction between desires and intentions, as they appear to be two separate constructs in behavioural models (Carrus et al. 2008).

With regards to its relevance to environmental choice the study of 'desires' may help to explain the intention-behaviour gap. It is possible that one may desire to act more sustainably, but lack the behavioural intent to translate desires into action. Indeed a lack of 'implementation intentions' (i.e. behavioural options) has been used to explain the intentionbehaviour gap (Carrington et al. 2010). It is possible that previous TPB research on sustainable choice has perhaps measured 'desires' rather than intentions, meaning that individuals seek to increase PE behaviour but do not know how they can do this. Thus, the intention-behaviour gap may more accurately reflect a desire-behaviour gap. Alternatively, it may be that concrete 
knowledge linked to behavioural intentions is not enough to motivate behaviour change; individuals must also desire behaviour change. For example, you may know that using the train to commute to work is more environmentally friendly than driving to work, yet you do not possess the desire to change your behaviour. The addition of desire as a mediator/moderator to planned behaviour change related to environmental choice thus offer an intriguing avenue for future research on the intention-behaviour gap.

Another important addition of the MGB is the role of emotional processing when making decisions about sustainable behaviour. Emotions are defined as multi-attribute states that are associated with important goals (Oatley, Keltner and Jenkins 2006), meaning that individuals can experience both positive and negative emotions at the same time, linked to one's goals. For example, when considering whether to take public transport to work in order to reduce one's carbon emissions, one may anticipate 'pride' for having achieved a sustainable goal but also anticipate potential regret if public transport is delayed making one late for work. 'Emotion' is distinct from 'affect' as it is context-dependent and may occur for a limited time only, whereas affect reflects more stable and broad-based dispositions linked to mood and feelings towards a stimulus that remain relatively stable over time (Koenig-Lewis et al. 2014). The anticipation of guilt for not recycling one's waste may be reduced if the individual believes that they have engaged in compensatory green behaviour by taking the bus to work; emotions are context and situation dependent. Alternatively, one's 'emotional affinity' to nature, an affective state, is more stable over time. Indeed, one's affective connectedness to nature is associated with childhood upbringing whereby rural dwellers have been found to feel more connected to nature and so have greater PE intentions and behaviours (Hinds and Sparks 2008). This is an important distinction in the literature whereby affect is a broad-based state linked to individual differences whereas emotion is more variable and context-dependent. As environmental decision making has been found to vary according to context, with individuals making PE choices for one behaviour (e.g. recycling) but not for another (e.g. purchase of low carbon products) (Szmigin, Carrigan and McEachern 2009), then emotions may act as important context-dependent predictors for adopting different types of PE behaviours.

The MGB is thus a useful extension to the TPB due to the incorporation of emotional weights, especially when exploring emotionally salient issues such as global warming. Indeed, some authors argue that emotions are more important than cognitions when formulating attitudes about environmental issues due to this emotional salience (Pooley and O'Connor 2000). Much of the past literature on emotions and decision-making has tended to focus on 
negative emotions as more influential for choice than positive emotions (Richard et al. 1996). This links to the heuristics and biases literature on 'loss aversion', which suggests that individuals will make larger efforts to avoid losing than they would to achieve similar gains (Tversky and Kahneman 1974). Indeed, Elgaaied (2012) found that anticipated guilt for not recycling appeared to motivate individuals to adopt recycling behaviour, and that the anticipation of negative emotions was more influential than the anticipation of negative rational consequences (i.e. 'it is bad for the planet if you don't recycle'). Anticipated negative emotions have been linked to increased recycling intentions and the use of greener modes of transport (Carrus, et al. 2008), with specific emotions such as guilt and embarrassment playing key roles (Kaiser, Schultz, Berenguer, Corral-Verdugo and Tankha 2008). Although less well explored, the anticipation of positive emotions has also been associated with PE choice. It has been found that feelings of pride when one's social group acted in a sustainable way led to greater in-group favouritism and feelings of superiority (Harth, Leach and Kessler 2013). Furthermore, in a study on consumer behaviour it was found that the anticipation of positive emotions had a larger effect on behaviour than negative emotions (Koenig-Lewis et al. 2014). This suggests that interventions to increase PE behaviour should focus on the positive emotional benefits one will experience following PE behaviour change rather than negative emotions for not acting in a sustainable manner. The incorporation of emotion into models predicting PE intentions and behaviour increases explained variance in comparison to TPB up to as high as $61 \%$ (KoenigLewis et al. 2014) and so it is important to explore their role in designing interventions.

A limitation with previous research on emotions and sustainable decision-making relates to the methodological selection of emotions for empirical study. Indeed, research has tended to study the effects of a single emotion on decision-making, such as guilt (e.g. Elgaaied 2012) rather than exploring a wide range of both positive and negative emotions. Furthermore, when studies do incorporate a wider range of emotions, the selection of these emotions for incorporation into the study is often relatively vague and ad-hoc, based upon a sample of emotions that have been studied in the previous literature on general emotional regulation (e.g. Carrus et al. 2008). As emotions are context-dependent and variable, this therefore creates methodological issues with regards to the scientific basis for the selection of different emotions in studies that are specifically designed to investigate environmental choice. As such, a key priority for this paper will be to explore the different types of positive and negative emotions that are generated by participants when thinking about environmental behaviour. Indeed this is a step that is recommended by Ajzen (2002) for any research that seeks to build upon the TPB. 
It is hoped that the results from this type of approach could provide a foundation upon which future studies might potentially build when investigating the role of emotions in environmental choice.

\section{Method}

This paper sought to explore participants' free-recall responses to an online survey about 'pro-environmental' (PE) behaviour. This was to explore how people actually think about global warming by asking them to generate their own responses to a series of open questions, rather than collecting data on how people believe they ought to think in response to primed statements. Previous research has tended to investigate PE behaviour by presenting vignettes or asking participants to rank their attitudes or estimate the likelihood that they will perform specific types of behaviour, such as recycling or using public transport. However, this methodology generates findings that consistently suggest there is a gap between PE intentions and behaviour (Bamberg 2003), whereby individuals state they will act in a certain way but fail to do so. It is possible that this is because people do not readily think about these behaviours on a day-to-day basis. For example, someone who is asked whether they would buy low carbon products in a supermarket may agree that they would, but when asked to think about different types of PE behaviour they may not report this type of behaviour because it is not actually salient to them. That might well be the reason that they fail to think about buying low carbon products when shopping. Using open questions that ask participants to generate beliefs and thoughts about sustainable behaviours may provide a more accurate reflection of how people think about global warming and sustainable behaviour in their everyday lives.

The primary purpose for this study was, therefore, to provide a qualitative exploration of how people think about and conceptualise PE behaviour using a series of open-ended questions exploring: (i) different types of identified behaviours; (ii) perceived advantages/disadvantages for adopting PE behaviours; (iii) perceived social norms relating to PE behaviour; (iv) perceived control over one's ability to adopt PE behaviours; (v) anticipated emotional outcomes associated with PE behaviours; and (vi) emotions when comparing one's own to others' PE behaviours. These questions were designed based on previous variables identified by the TPB (Ajzen 1992) and the MGB (Perugini and Bagozzi 2001) to ensure a comprehensive exploratory approach. This study was conducted in accordance with the British Psychological Society's Code of Ethics and Conduct, and approved by the Departmental Research Ethics Committee (DREC) at Edge Hill University. 


\subsection{Participants}

A total of $n=61$ participants were recruited via social media (Facebook, Twitter) and took part in the online survey (one participant had to be excluded because of missing data). Of these, 21 were male and 39 were female. Ages ranged between 18 and 58, with a mean age of 25.64 years $(\mathrm{SD}=9.51)$.

\subsection{Materials}

Participants completed an online survey (using the Bristol Online Survey) that consisted of 19 questions in total, which took approximately 15 minutes to complete. The survey was split into eight sections as follows.

\subsubsection{Demographic information}

Participants were asked to state their age, sex and geographic living location (i.e. rural, suburban, urban).

\subsubsection{Environmental attitudes and behaviour}

Participants were asked to rank their level of agreement using a Likert Scale ranging from 1 (completely disagree) to 7 (completely agree) on three items measuring their attitudes and behaviours. Environmental attitude was measured with two items: "I care about the environment"; "I am concerned about global warming" and PE behaviour was measured using one item: "I take active steps to try and reduce my carbon footprint".

\subsubsection{Types of PE Behaviours}

This section sought to explore the different types of PE behaviours that were generated by participants. Participants were asked one open question: "Think about different types of environmentally friendly behaviour. Consider any behaviours that you do currently or ones that you might be able to start doing in the next month. Please list all types of behaviour that come to mind. Examples might be cycling to university/work, or recycling household waste."

\subsubsection{Behavioural Outcomes}

Participants were asked to think about the possible advantages and disadvantages for adopting PE behaviour. This was to measure their perception of the potential 'behavioural outcomes' that may arise and provide information about potential reasons both for and against adopting PE behaviour: "What do you see as the advantages of adopting environmentally friendly behaviours?"; "What do you see as the disadvantages of adopting environmentally friendly behaviours?" 


\subsubsection{Control Beliefs}

Participants were also asked about how much control they perceived to have over their ability to adopt PE behaviours using two items: "Please list any factors or circumstances that would make it easy or enable you to act in an environmentally friendly way"; "Please list any factors that would make it difficult or prevent you from acting in an environmentally friendly way".

\subsubsection{Social Referents}

Four items were used to explore how participants thought about PE behaviour with regards to social referents and social norms. Items included: "Please list the individuals or groups who might approve of you adopting more environmentally friendly behaviours"; "Please list the individuals or groups who might disapprove of you adopting more environmentally friendly behaviours"; "Sometimes we look to other individuals or groups when we are not sure what to do. Please list the individuals or groups who you think are most likely to perform environmentally friendly behaviours"; "Please list the individuals or groups who are least likely to perform environmentally friendly behaviours".

\subsubsection{Emotional Outcomes}

Two items were also included to measure one's beliefs about anticipated emotional outcomes following the success or failure to adopt PE behaviours: "Imagine that you have successfully changed your behaviour to be more environmentally friendly in the past month. Please list as many different feelings and emotions that you might feel as a result of this"; "Imagine that you have failed to change your behaviour to be more environmentally friendly over the past month. Please list as many different feelings or emotions that you might feel as a result of this".

\subsubsection{Social-Emotional Referents}

In addition to anticipated emotions, the study also sought to see how emotions interacted with social comparison. The following two items measured this: "Think about the individuals and groups who you think would approve of you adopting more environmentally friendly behaviours. Please list as many different feelings and emotions that you might feel if they successfully adopt more environmentally friendly behaviours but you do not"; "Please list 
as many different feelings and emotions that you might feel if you successfully adopt more environmentally friendly behaviours but they do not".

\subsection{Data analysis}

Data was analysed with a mixed methods approach. Participants' initial responses to the open-text questions were analysed using thematic analysis. Thematic analysis is a form of qualitative analysis that combines both inductive (bottom-up) and deductive (top-down) techniques (Braun and Clarke 2006). This process involves the initial reading of participant responses to each question and using an inductive approach to note down emerging 'themes' or patterns in the data. Once initial wider coding has been carried out, it is then possible to refine and collapse themes into sub-themes under larger thematic headings. For example, when participants were asked about possible PE behaviours they may adopt, the identification of using more sustainable 'lighting', 'electricity', 'water' and 'home heating' were sub-themes under the larger thematic heading of 'home energy consumption'. This process involves repeated reading and cross-referencing between themes and participant responses to refine thematic categories into meaningful components. Once themes are identified and clearly defined, it is then possible to return to the data and use deductive coding to search for additional utterances that can be categorised into a theme but may have been missed during initial coding, using a 'coding dictionary' that defines each theme. This second phase allows the data to be analysed using content analysis, whereby the frequencies of different themes within the data set are statistically analysed for frequencies (Ryan and Bernard 2000). This allowed for further analysis of the data to see how themes interacted with one another. It is important when performing frequency analyses of qualitative data that inter-rater reliability is assessed for objectivity. Cohen's kappa analyses in this area indicated excellent inter-rater reliability between two raters, $k=.833, p<.001$.

\subsection{Procedure}

Participants were recruited via social media (Facebook, Twitter) and asked if they would like to participate in a 15-minute survey exploring perceptions of pro-environmental behaviour and sustainability. They were provided with a link to the online survey, which was designed using the 'Bristol Online Survey'. The survey presented participants with an information sheet that explained the aims of the survey. If they agreed to participate, the clicked a box to indicate their consent and completed the questions. Data was analysed with assistance from the qualitative data analysis tool 'NVivo' and quantitative data analysis tool 'SPSS'. 


\section{Results}

\subsection{Environmental concern and behaviour}

A paired samples t-test found that participants had significantly higher environmental concern scores $(\mathrm{M}=5.52, \mathrm{SD}=1.36)$ than environmental behaviour scores $(\mathrm{M}=4.52, \mathrm{SD}=1.57)$, $t(59)=6.80, p<.001$. This suggests an awareness of the disconnect between concern and behaviour as participants were aware that they did not take active steps to try to reduce their carbon footprint despite being concerned about the environment and global warming.

\subsubsection{Environmental concern/behaviour, age and gender}

There were no significant differences between genders for either environmental concern, $t(57)=-.197, p=.844$, or behaviour, $t(57)=.915, p=.364$. However, age was significantly and positively correlated with environmental concern, $r=.320, p=.013$. Although environmental behaviour was also positively correlated with age, the relationship was not significant, $r=.140$, $p=.287$.

\subsubsection{Environmental concern/behaviour and dwelling location}

Participants were asked to identify whether they lived in a rural, suburban or urban environments. A one-way ANOVA found no significant relationship between dwelling location and environmental concern, $F(2,59)=.225, p=.799$, or dwelling location and behaviour, $F(2,59)=.956, p=.390$. However, the means did indicate a pattern for both environmental concern and behaviour in that rural dwellers had the highest scores on both, followed by suburban and finally urban dwellers (Table 1).

Table 1: Means and standard deviations for environmental concern and behaviour for rural, suburban and urban dwellers

\begin{tabular}{|l|l|l|l|}
\hline & Rural & Suburban & Urban \\
\hline Environmental concern & $5.90(.961)$ & $5.46(1.49)$ & $5.50(.864)$ \\
\hline Environmental behaviour & $4.80(1.30)$ & $4.62(1.66)$ & $3.90(1.10)$ \\
\hline
\end{tabular}

\subsection{Types of PE behaviours}

A thematic analysis of participant responses when asked to identify "different types of behaviours that you could adopt to help protect the environment" is displayed in Figure 1. It 
was themed into five categories: (i) home energy consumption; (ii) transport behaviour; (iii) purchasing behaviour; (iv) food behaviour; and (v) general sustainable behaviours.

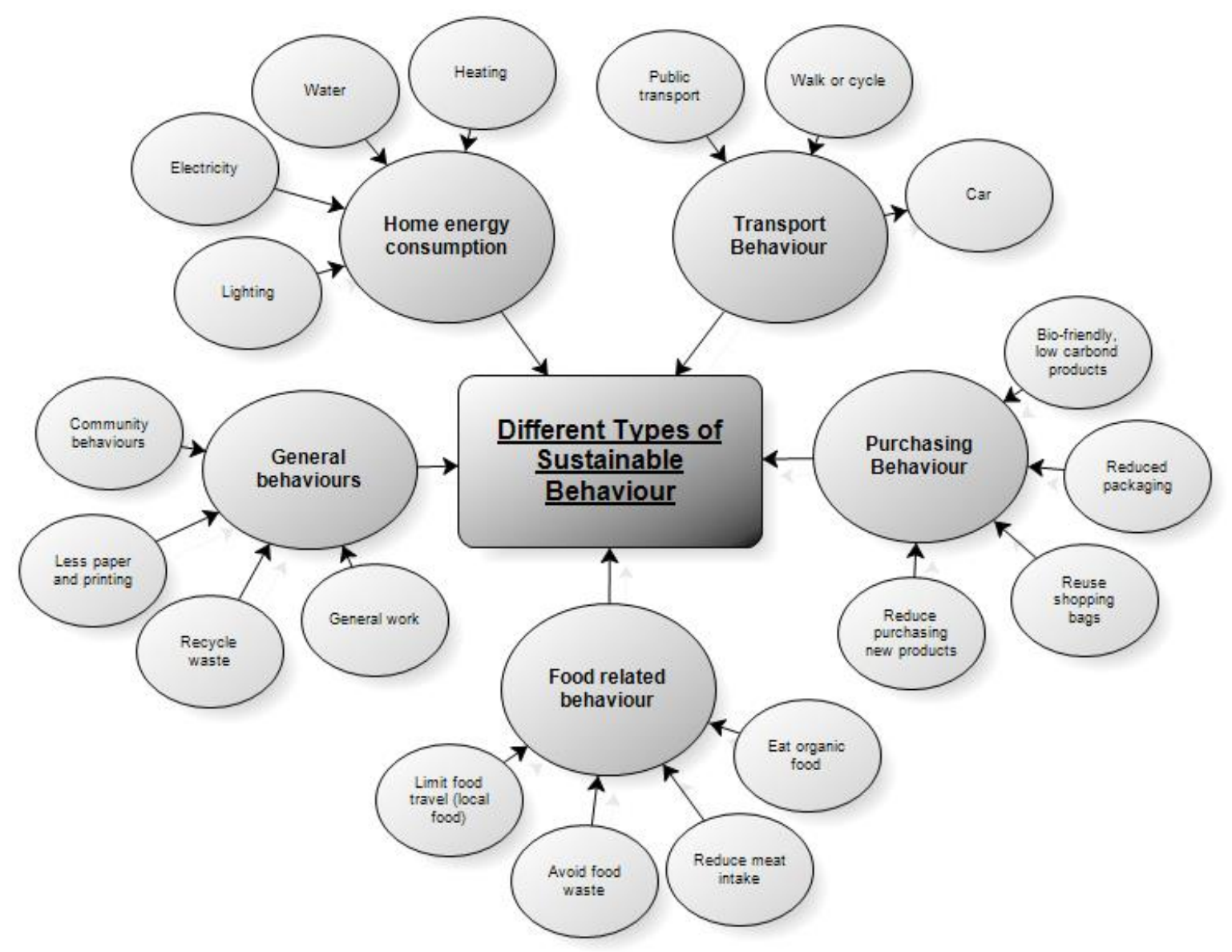

Figure 1: A typology of behaviours associated to sustainability

Home Energy Consumption reflected ideas about increasing the efficiency of home energy use, linked to water, heating, electricity and/or lighting: "energy efficient measures are used such as insulation and being energy conscious"; "unplug/switch off plug socket for electronics when not in use". Transport Behaviour was linked to a desire by participants to reduce their reliance on non-green transport such as personal cars: "become less dependent on the car, especially over short distances", and instead adopting more eco-friendly transport behaviour linked to using public transport or no emissions through walking or cycling: "using public transport when possible and even better, walking or cycling when possible".

Participants identified four ways that they could adopt more sustainable purchasing behaviour by: (i) purchasing bio-friendly or low-carbon products: "Buying bio-friendly products (cleaning chemicals, cosmetics...)"; (ii) reducing overall purchasing and only buying necessary or recycled products: "avoid clothes waste - try to reuse clothes or donate to 
others"; (iii) to reuse shopping bags: "always bring bags when you go shopping”; and (iv) to buy products with less packaging or remove it where possible: "insist on supermarkets removing all food packaging when purchasing". Food-related behaviour was linked to avoiding overall food waste: "not buying excess food that goes to waste" and making food choices that are more sustainable, such as reducing meat consumption or becoming vegetarian/vegan: "mostly choosing vegetarian options in restaurants", favouring organic and ethically sourced products: "buy meat from 'trusted' sources (i.e. from a local butcher who does not import any food for the animals)", and limiting the distance that food has travelled by buying locally: "only buying food that is locally produced".

A final category of sustainable behaviour types refers to more general behaviours that are relatively abstract and reflect general ways one may adopt sustainable choices in everyday life, such as: recycling waste: "recycling and separating waste - paper, plastic etc."; using less paper and printing whenever possible: "only print when necessary - paper free where possible"; making sustainable choices in the workplace: "increase environmental consciousness at work"; and adopting social or community focussed behaviours to promote sustainability in the local area through behaviours such as not dropping litter and general "Spreading awareness to others".

\subsubsection{Frequency analyses of the different types of $P E$ behaviours identified by participants}

As indicated in Figure 2, the largest category of behaviours was general sustainable behaviours as $91.5 \%$ identified this type of behaviour as a way they could act in a more sustainable way. This was followed by transport-related behaviour (86.4\%), home energy behaviour (72.9\%), purchasing behaviour (42.4\%) and food-related behaviour (40.7\%). 


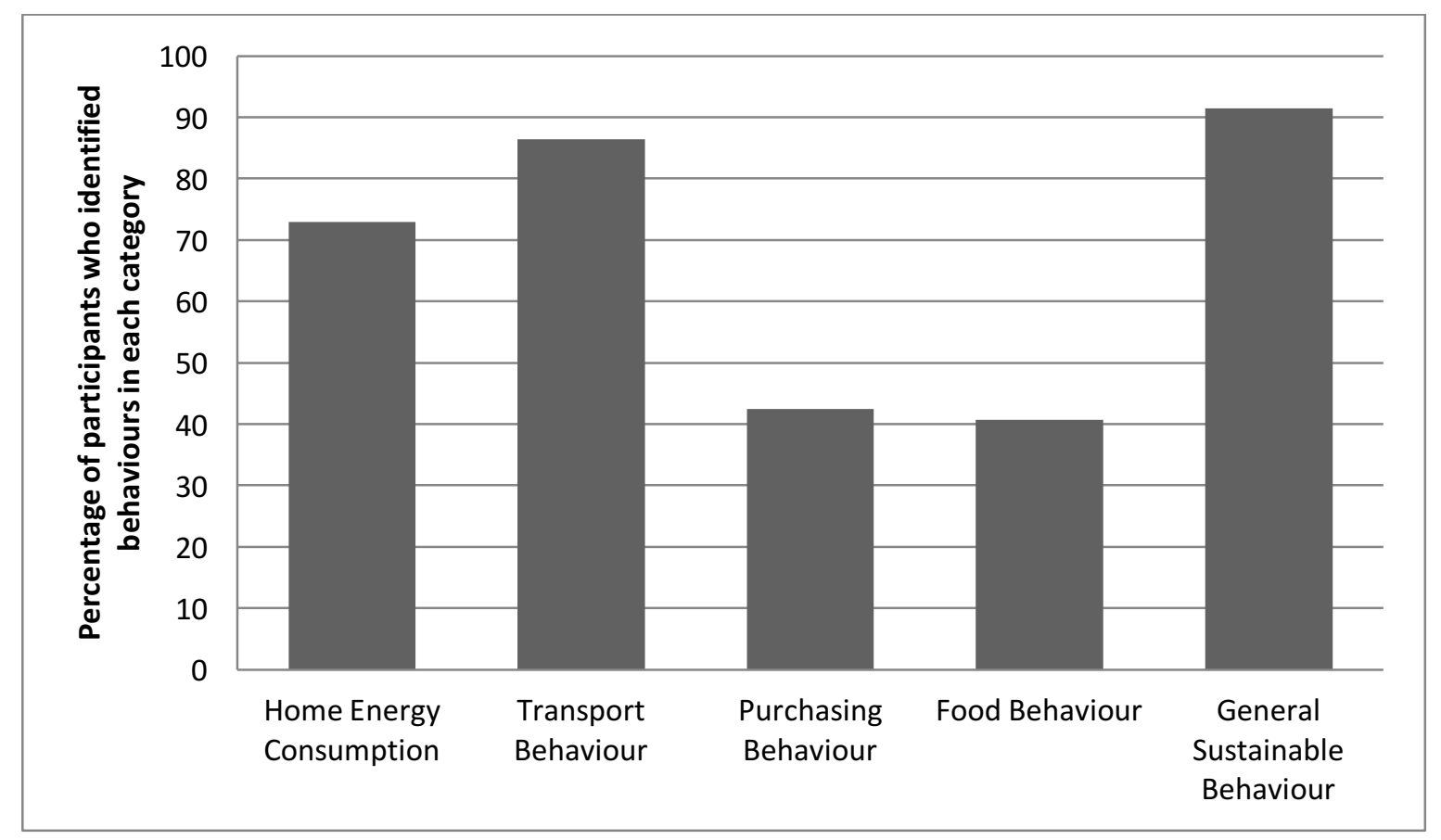

Figure 2: Percentage of participants who identified sustainable behaviours within each category

The mean average number of behaviours identified by participants was $5.31(\mathrm{SD}=2.68)$ out of a possible 18. A Pearson's correlation found that there was a significant positive correlation between environmental concern and the number of identified behaviours, $r=.463$, $p<.001$. There was also a significant positive correlation between scores for environmental behaviour and the number of behaviours identified, $r=.296, p=.024$. There was also found to be a significant interaction between the number of identified behaviours and dwelling location, $F(2,56)=3.683, p=.031$. Post-hoc tests indicated that this was due to the difference between rural and urban dwellers, whereby urban dwellers $(\mathrm{M}=3.70)$ identified significantly fewer behaviours than rural dwellers $(\mathrm{M}=7.40), p=.029$.

\subsubsection{The likelihood of identifying different PE categories}

The mean number of categories identified by participants was $3.40(\mathrm{SD}=1.11)$ out of a possible five. A median split on age found that older participants (i.e. age $>25 y e a r s$ ) were 3.58 times more likely to identify PE behaviours specifically associated with food in comparison to younger participants, $\chi^{2}=5.545, p=.019$. It was also found that those who scored high on 'environmental concern' were 6.52 times more likely to identify food as a category of PE behaviour, $\chi^{2}=10.309, p=.001$ and 2.88 times more likely to identify purchasing behaviour as a type of PE behaviour, yet this was marginally outside the range of significance, $\chi^{2}=3.55$, $p=.059$. This suggests that the category relating to 'food behaviour' is unique, as it tends to 
only be identified by participants that are older or have higher scores on EC. This category may therefore reflect more specialist knowledge, which has been gained either through experience as a consumer or by those who are concerned about the environment and so may be more aware of different ways to act in a sustainable way.

\subsection{Perceived behavioural outcomes for adopting sustainable behaviour}

\subsubsection{Advantages}

Participants' perceived advantages for adopting PE behaviour were themed into two main categories that reflected 'distant-future advantages', which focussed on long-term and abstract benefits to PE behaviour and 'near-future advantages', which were oriented around immediate gains (Figure 3). Distant-future advantages included: (i) protecting biodiversity and wildlife; (ii) protecting the earth's resources; (iii) making a better world for future generations; (iv) protecting against and preventing climate change; and (v) helping less affluent countries who will be affected by global warming. Near-future advantages were further sub-themed into two categories: (i) lifestyle benefits; and (ii) psycho-social benefits. Lifestyle benefits included having: (i) a cleaner and better environment to live in and enjoy; (ii) health benefits from improved fitness; and (iii) saving money. Psycho-social benefits included: (i) an improved sense of wellbeing; and (ii) feeling a part of a collective social conscience.

Interestingly, although $43.9 \%$ of participants identified both near- and distant-oriented advantages for sustainable behaviour, $37.7 \%$ only identified distant future advantages compared to $15.8 \%$ who identified near-future advantages only. This suggests that over a third of participants only thought about distant and more abstract benefits for adopting PE behaviour. This has important implications for how people conceptualise PE behaviour as it would suggest that a large proportion only perceive distant and more abstract benefits to adopting behaviour. A greater focus on near-future benefits (e.g. lifestyle benefits) could offer a potential opportunity for intervention. 


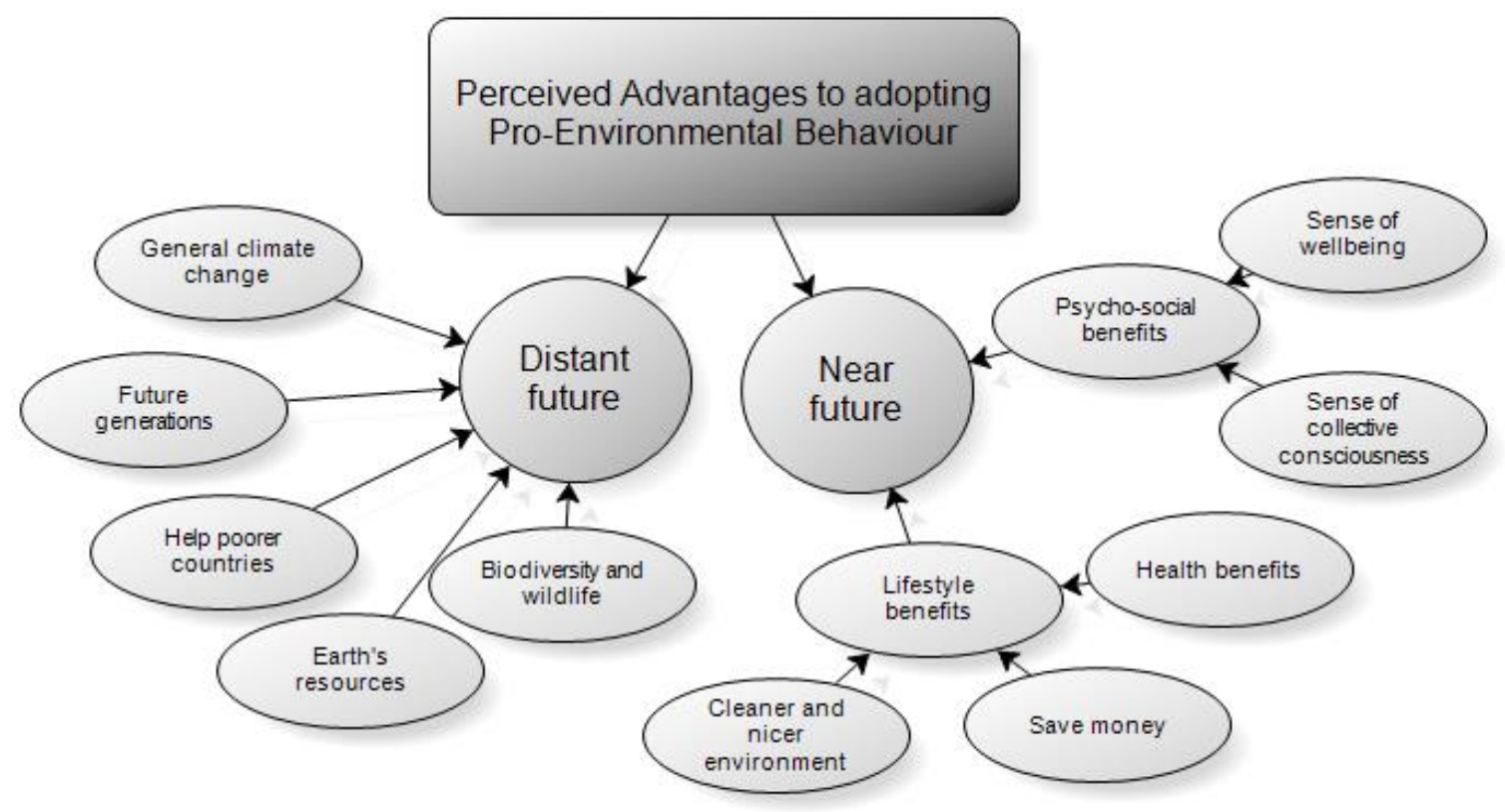

Figure 3: The perceived advantages of adopting sustainable behaviour

\subsubsection{Disadvantages}

The perceived disadvantages of adopting PE behaviour were themed into seven categories, under two thematic headings: (i) practical barriers (lower quality of life, monetary demands, time demands); and (ii) psychological barriers (effort, hard to change behaviour, inconvenience and the view that there was no point) (Figure 4).

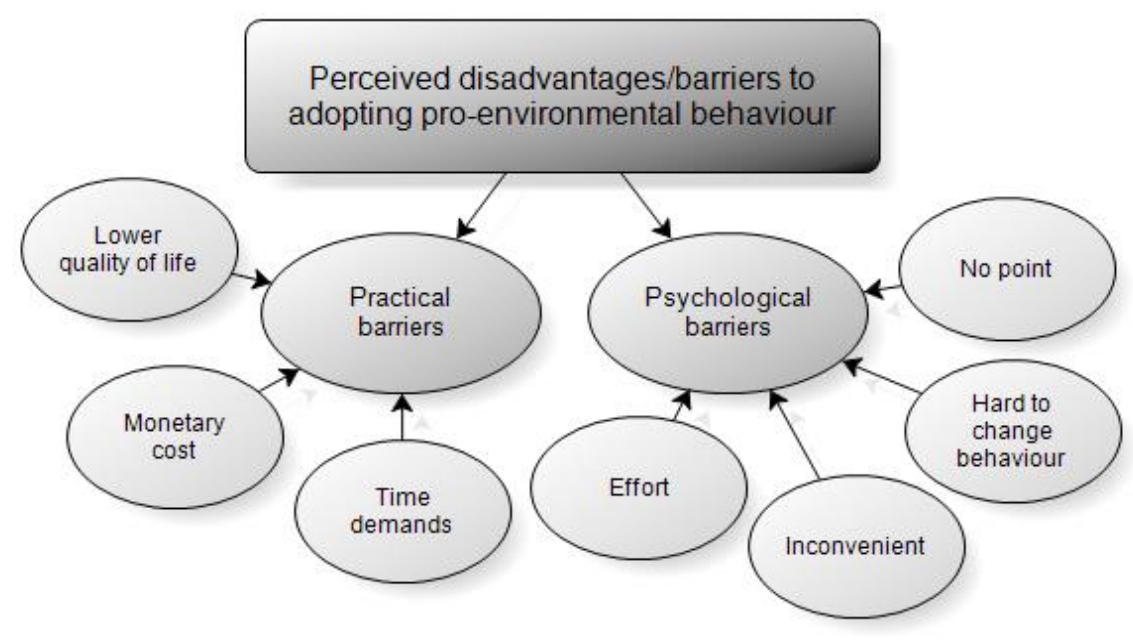

Figure 4: Participants' perceived disadvantages/barriers to adopting PE behaviour

As displayed in Figure 5, the most commonly perceived barrier to adopting PE behaviour was due to 'time demands' $(45.5 \%)$, followed by effort $(29.1 \%)$, inconvenience and money 
(27.3\%), difficulty in changing behaviour (16.4\%) and the belief that there was no point in adopting sustainable behaviours (7.3\%). In addition, $7.3 \%$ of participants stated that they perceived no disadvantages to adopting PE behaviour.

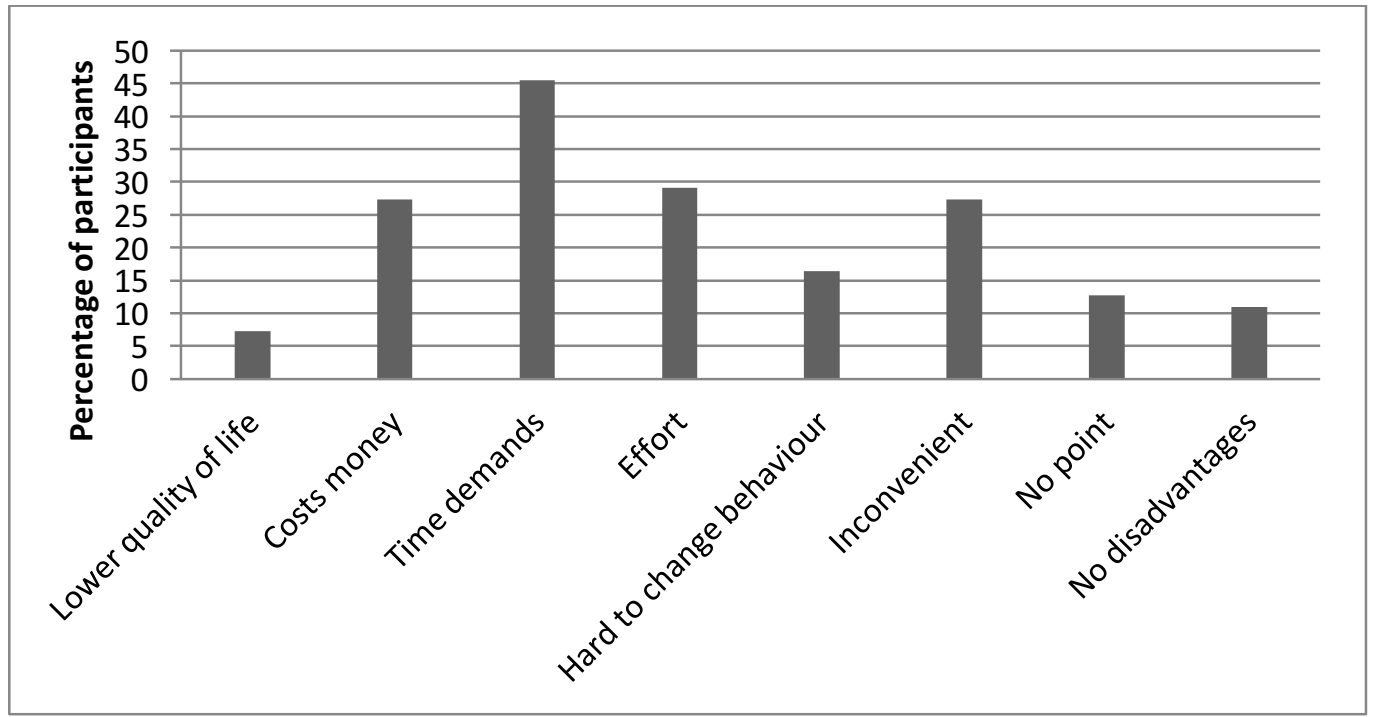

\section{Figure 5: Percentage of participants who identified each disadvantage}

A set of correlations were carried out to determine whether there was a relationship between the number of identified advantages and disadvantages to behaviours and environmental concern and behaviour. There were found to be no relationship between environmental concern and the number of advantages identified by participants, $r=.210$, $p=.117$, or number of disadvantages identified by participants, $r=.092, r=.505$. There was also no relationship between scores for environmental behaviour and the perceived number of advantages, $r=.074, p=.586$, or disadvantages, $r=-.052, p=.708$. There was, however, a significant positive correlation between the total number of behaviours identified by participants and the number of advantages identified, $r=.530, p<.001$, and the perceived number of disadvantages, $r=.430, p=.001$. It is possible that this reflects a more flexible way of thinking about PE behaviour, whereby those who identify a number of different types of PE behaviour also identify lots of different advantages and disadvantages to performing these behaviours.

\subsection{Control beliefs}

Participants were asked to identify the types of things that would make it easier or harder to act in a sustainable way. There were eight different types of 'enabler' identified by 
participants (Figure 5). These included: (i) access to green facilities: "plenty of recycling bins available: paper, plastic, cardboard, glass”; (ii) high standard green facilities, which are convenient and reliable: "good trains services and frequent buses"; (iii) affordable green products: "affordable range of eco-friendly alternative products to what I usually buy"; (iv) government initiatives that encourage sustainable behaviour through incentives: "financial incentives to get solar panels" or deterrents/taxes: "if the choice was taken away to not act environmentally friendly e.g. fine if not recycled"; (v) green businesses and organisations that can increase exposure to green options: "conscientious business that the public consume from (e.g. supermarkets) that reduce packaging; oil producers that use sustainable products"; (vi) green technology that makes it easier to act in a sustainable way: "sensory lights that switch off after a period of time with no movement-you don't have to remember to switch them off yourself"; (vii) better education and information on how to be 'green': "good information about what I should and shouldn't do, including education in school"; and (viii) social support from others linked to feelings of collective social responsibilities and green social norms: "it's easier when everyone else around you is also behaving that way, they act as a reminder".

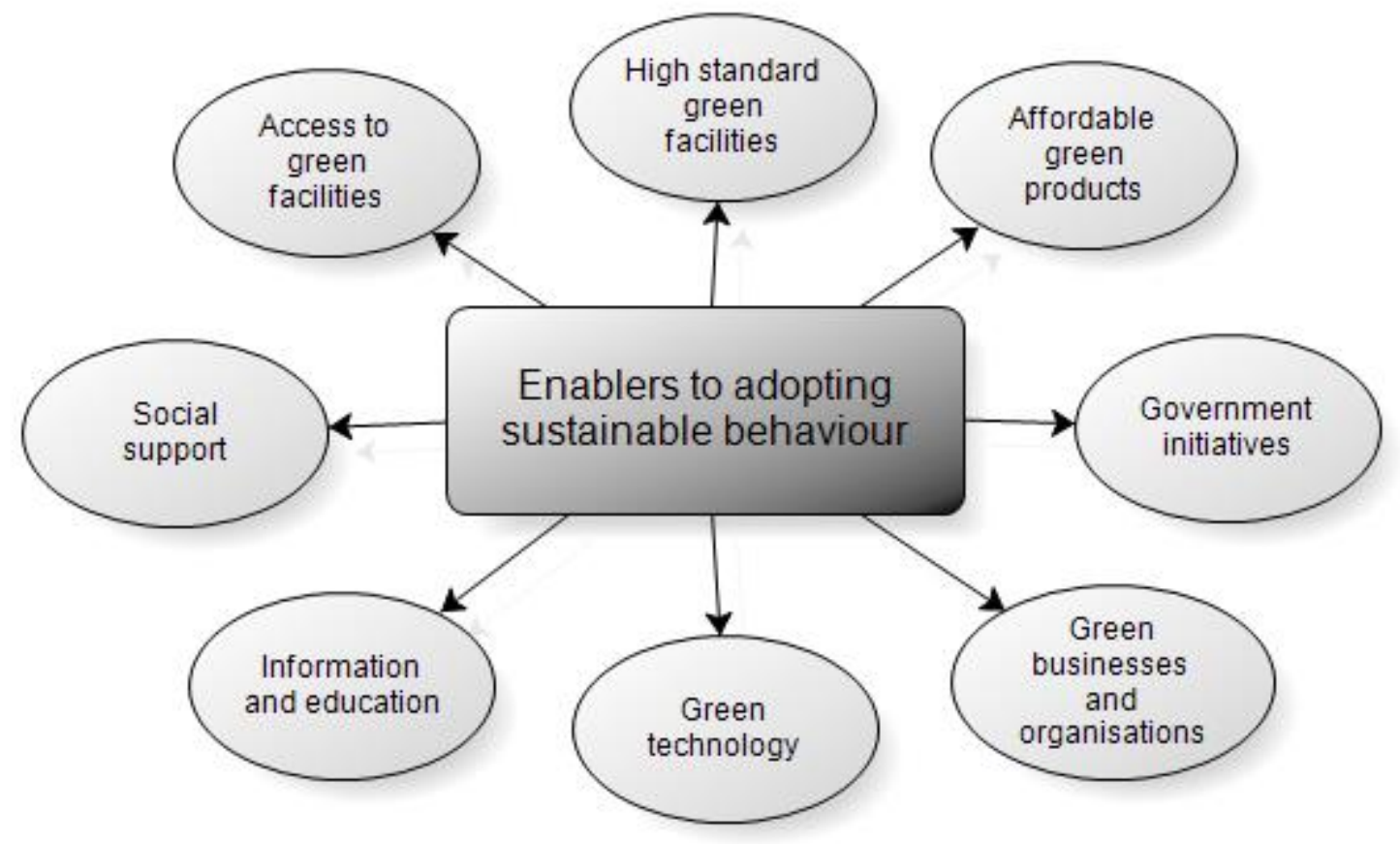

Figure 5: Factors that help to enable individuals to act in a more sustainable way

In terms of the percentage of participants who identified each 'enabling' category, it was found that $61.8 \%$ of participants stated that 'access to green facilities' would make sustainable behaviours easier, followed by access to information and education $(21.8 \%)$, 
government initiatives (20.0\%), high standard green facilities (18.2\%), affordable green products $(16.4 \%)$, greener businesses/organisations (12.7\%), social support (12.7\%) and green technology (5.5\%)(Figure 6).

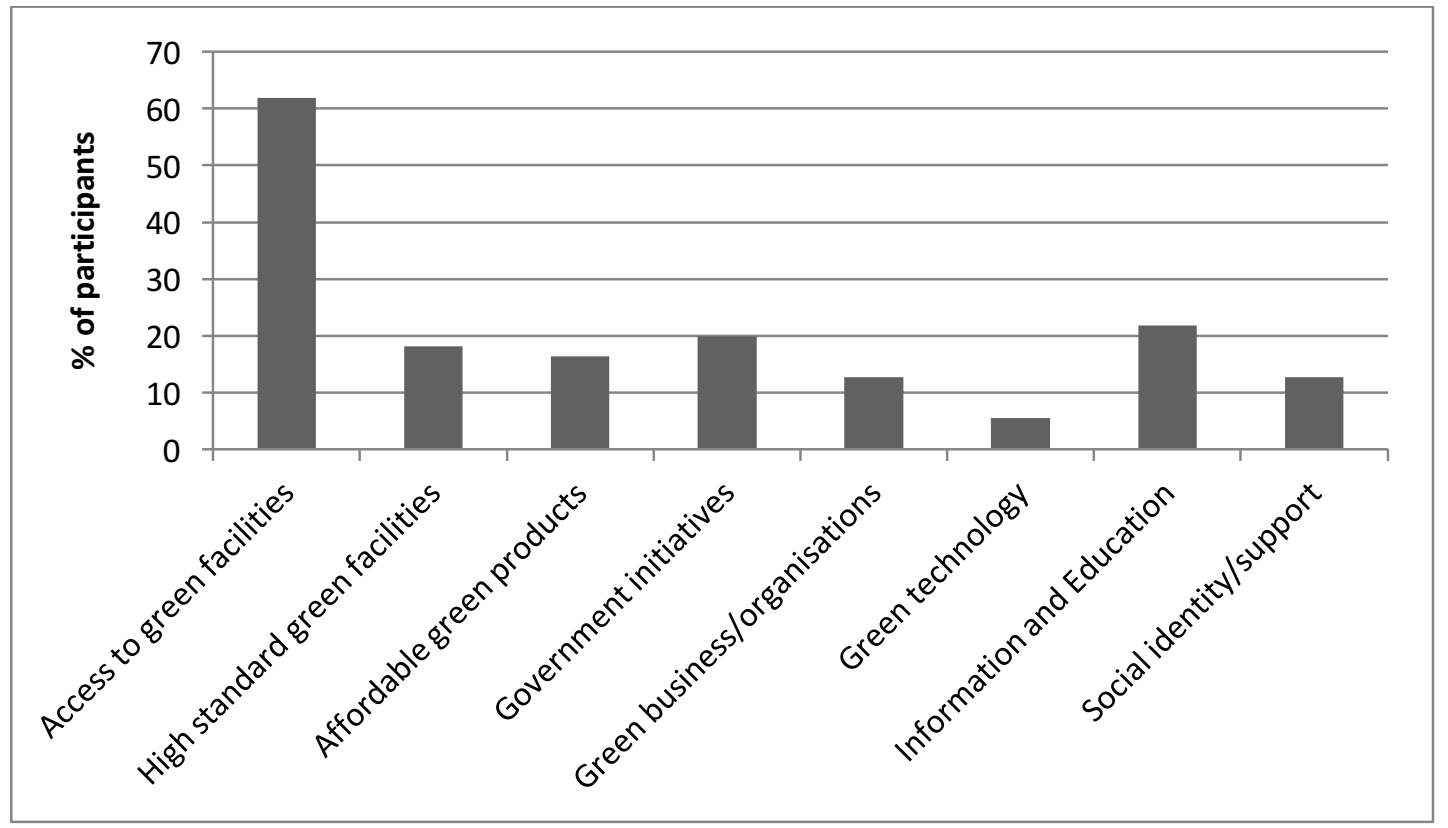

\section{Figure 6: Percentage of participants who identified each enabler}

Six themes were identified with regards to things that would make it more difficult for respondents to adopt PE behaviour (Figure 7). These were: (i) poor availability of green options: "no provision of external recycling bins (rented accommodation"; (ii) poor quality of green options: "public transport can be unreliable"; (iii) lack of commitment to act in a sustainable way: "lack of commitment in most cases"; (iv) additional cost, both monetary: "expensive environmentally friendly products" and in terms of added time to act in a sustainable way: "time is of the essence and acting in an environmentally friendly way would prove costly"; (v) lack of or confusing information on how to act sustainably: "lack of information on what could be done to help the environment"; and (vi) the prevalence of wasteful societal norms: "daily life has evolved around using oil and other harmful substances". 


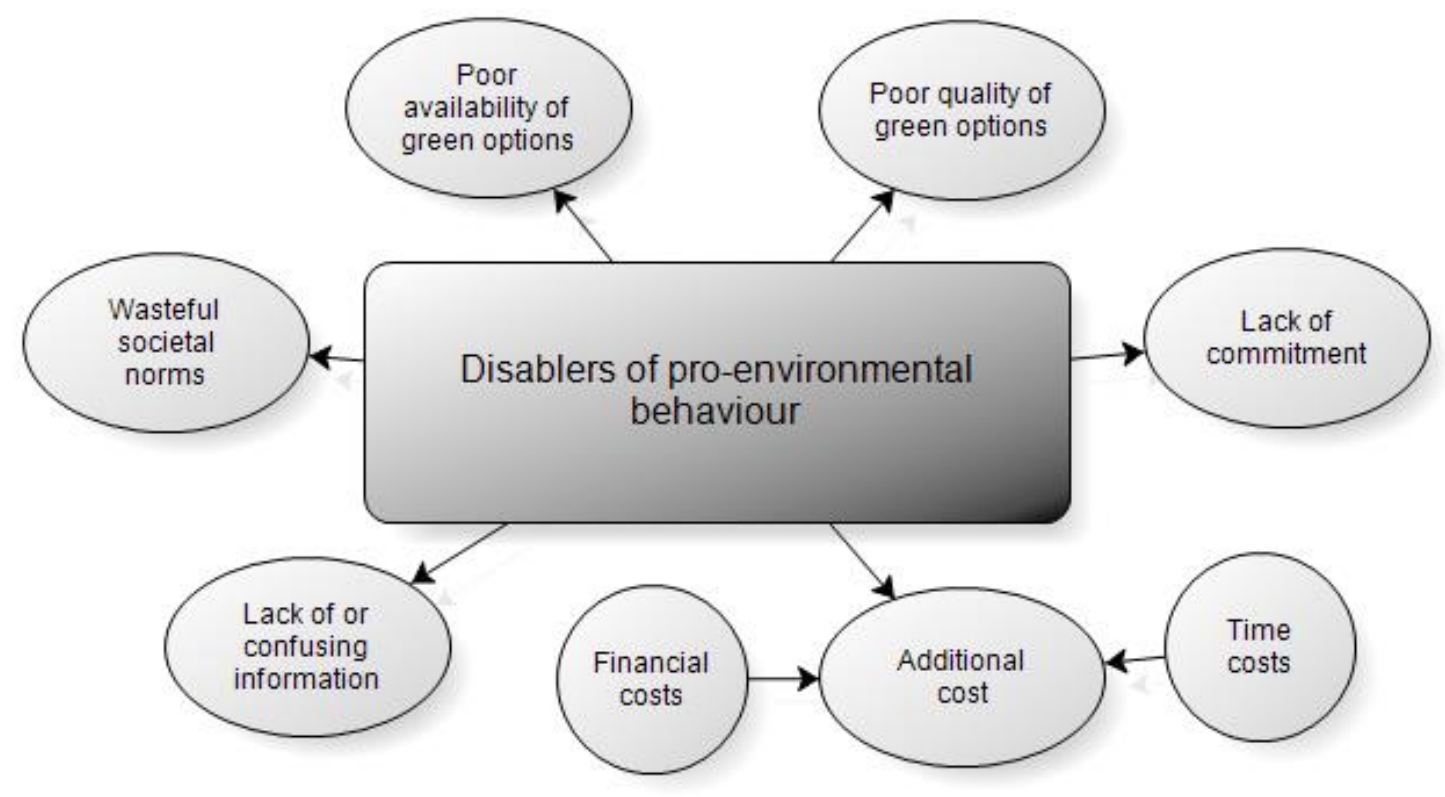

Figure 7: Things that make it less easy to adopt PE behaviour

The largest theme that was identified by participants was 'additional cost', which was identified by $50 \%$ of participants as a key barrier to PE behaviour (Figure 8). Within this category, $30 \%$ of all participants identified financial costs and $26 \%$ identified time costs. This was followed by lack of availability of green options (28\%), wasteful societal norms (26\%), lack of commitment (20\%), lack of or confusing information (18\%) and low quality of green options (12\%).

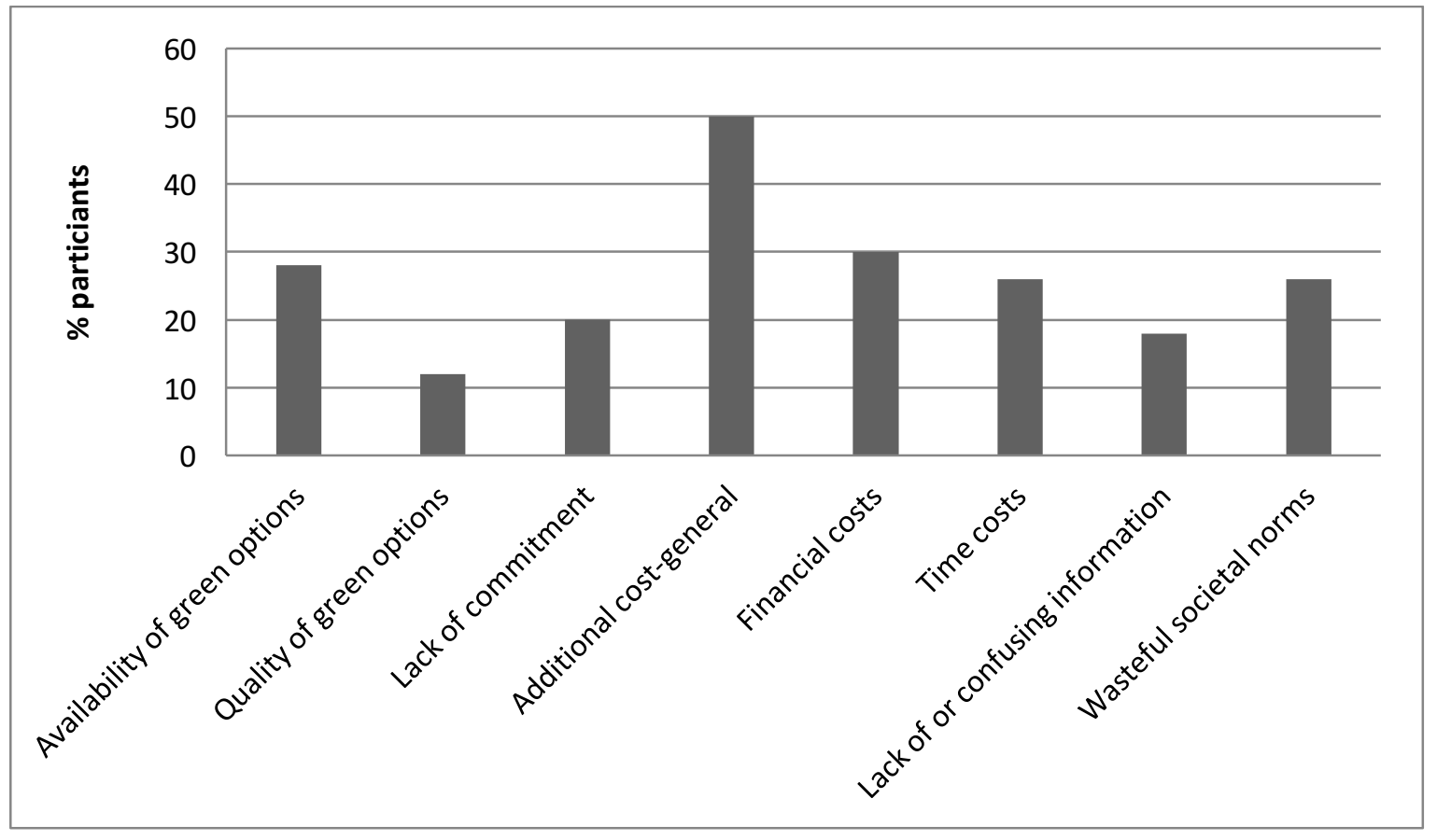


Figure 8: Percentage of participants who identified each category of 'disablers' to PE behaviour

\subsection{Social referents}

Participants were asked to identify "individuals or groups who may conduct sustainable behaviour themselves" and "others who may be supportive if you adopted pro-environmental behaviour". These questions were collapsed in coding to reflect one group of "proenvironmental social others' (Figure 9). There were four groups of social others: (i) green lobbyists, which included educators, environmentalists, public figures, the Green Party and charities who promote green behaviour; (ii) organisations, referring to businesses, government agencies and energy providers who supply or use green products; (iii) immediate social network, which refers to parents, family and friends who may act in a sustainable way; and (iv) wider social network, which include a variety of social others who may approve of adopting PE behaviour.

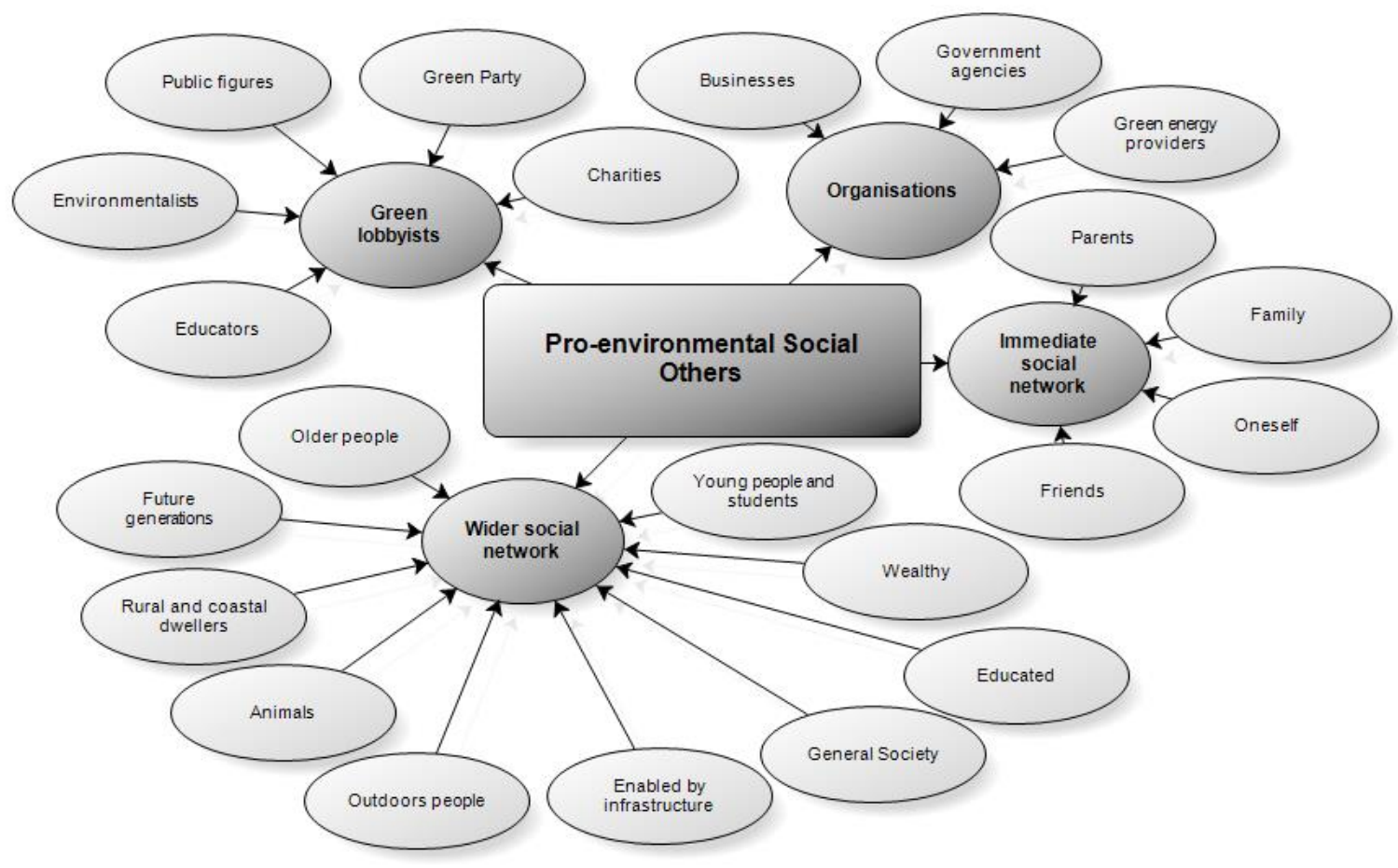

Figure 9: Social others identified by participants as being 'supportive' of PE behaviour

Participants were also asked to consider those who would be "unlikely to adopt sustainable behaviour themselves" or who may "disapprove of you adopting proenvironmentally behaviour". These were, as above, collapsed into one category to reflect social 
others who may be unsupportive of pro-environmental behaviour and themed into three categories: (i) organisations who invest in non-green products or may perceive PE behaviour as too costly, such as wasteful companies, profiteers (e.g. oil/gas industries), government and developing countries involved in industrialism (e.g. China); (ii) immediate social network, such as family and friends who are not interested in green behaviour; and (iii) wider social network who also disregard green behaviour. Furthermore, a number of participants expressed that they thought 'no-one' would be unsupportive of pro-environmental behaviour, which became a fourth category (Figure 10).

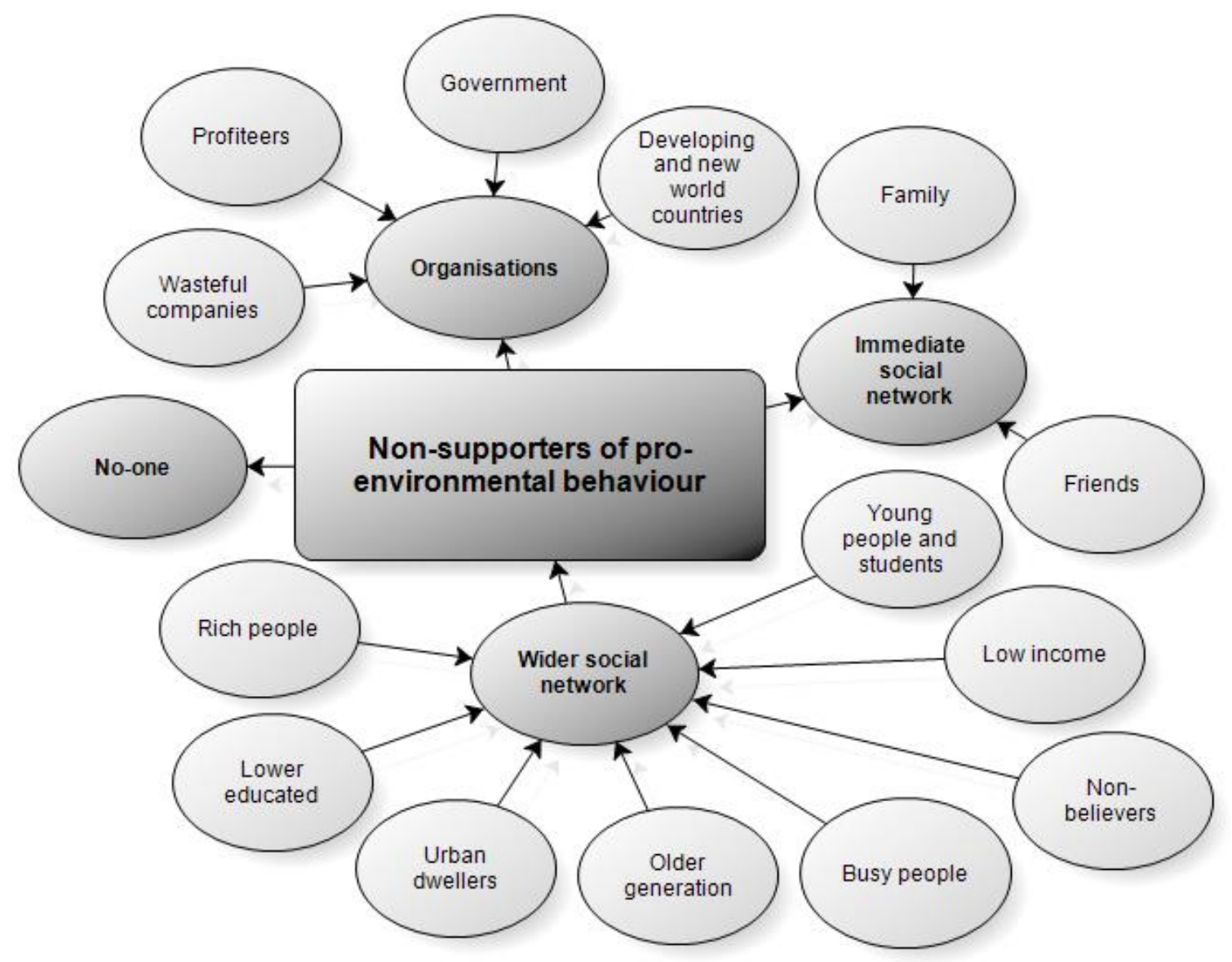

Figure 10: Social others identified by participants as being 'unsupportive' of proenvironmental behaviour

\subsection{Anticipated emotions}

\subsubsection{Positive emotions}

Participants were asked to think about the emotions they might feel if they adopted PE behaviour. As the research on emotional regulation with regard to sustainable behaviours is 
relatively limited, coding was deliberately kept as wide as possible to help inform further research. Figure 10 outlines the emotions that were themed into six categories. This shows that adopting sustainable behaviour might lead to: (i) positive self-identify, linked to feelings of well-being, happiness, relief and feeling uplifted and optimistic for the future: "I'd feel better about myself as a person"; (ii) a sense of personal pride, linked to pride and a sense of 'smugness' for having adopted PE behaviour: "would likely feel proud of myself for adopting these behaviours"; (iii) sense of leadership, which reflected feelings of empowerment, passion, influence, virtue, knowledge and motivation to help others change their behaviour for the better: "motivation to try and convince others of the necessity to change their behaviour"; (iv) sense of satisfaction, linked to contentment, fulfilment and satisfaction at changing their behaviour: "satisfaction with myself"; (v) a feeling of 'connectedness to others, associated with feeling one had contributed to society and part of a social movement: "glad to be playing my part; feel like I'm doing my bit"; and (vi) a sense of achievement, linked to feeling accomplished, successful and pleased with oneself: "achievement that I was helping the planet".

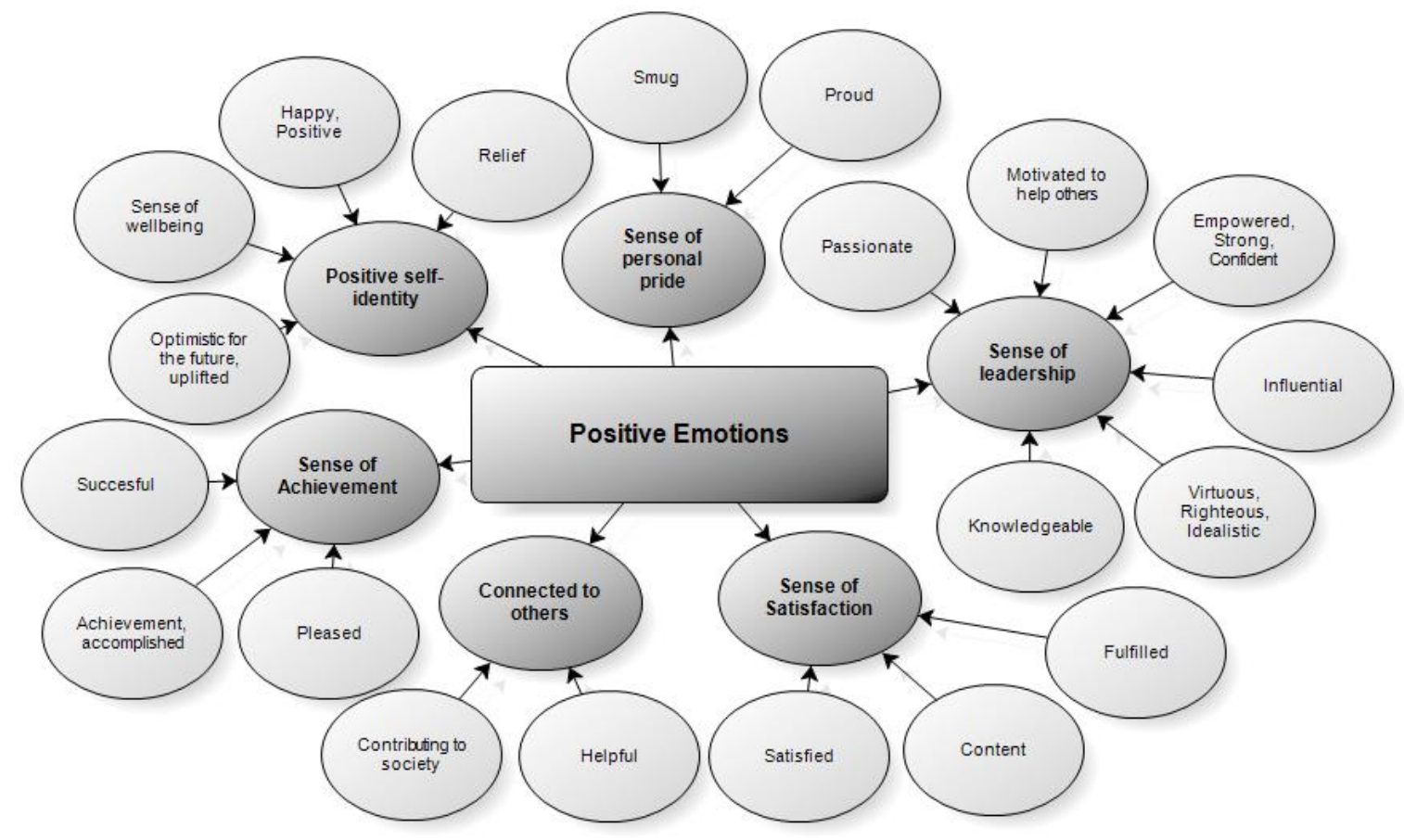

Figure 11: Positive emotions that participants anticipated they would feel if they successfully adopted PE behaviour 
In terms of the prevalence of these categories, $72.4 \%$ of participants anticipated feeling a positive self-identity, followed by pride (56.9\%), sense of connectedness (34.5\%), sense of achievement (32.8\%), satisfaction (25.9\%) and sense of leadership (24.1) (Figure 12).

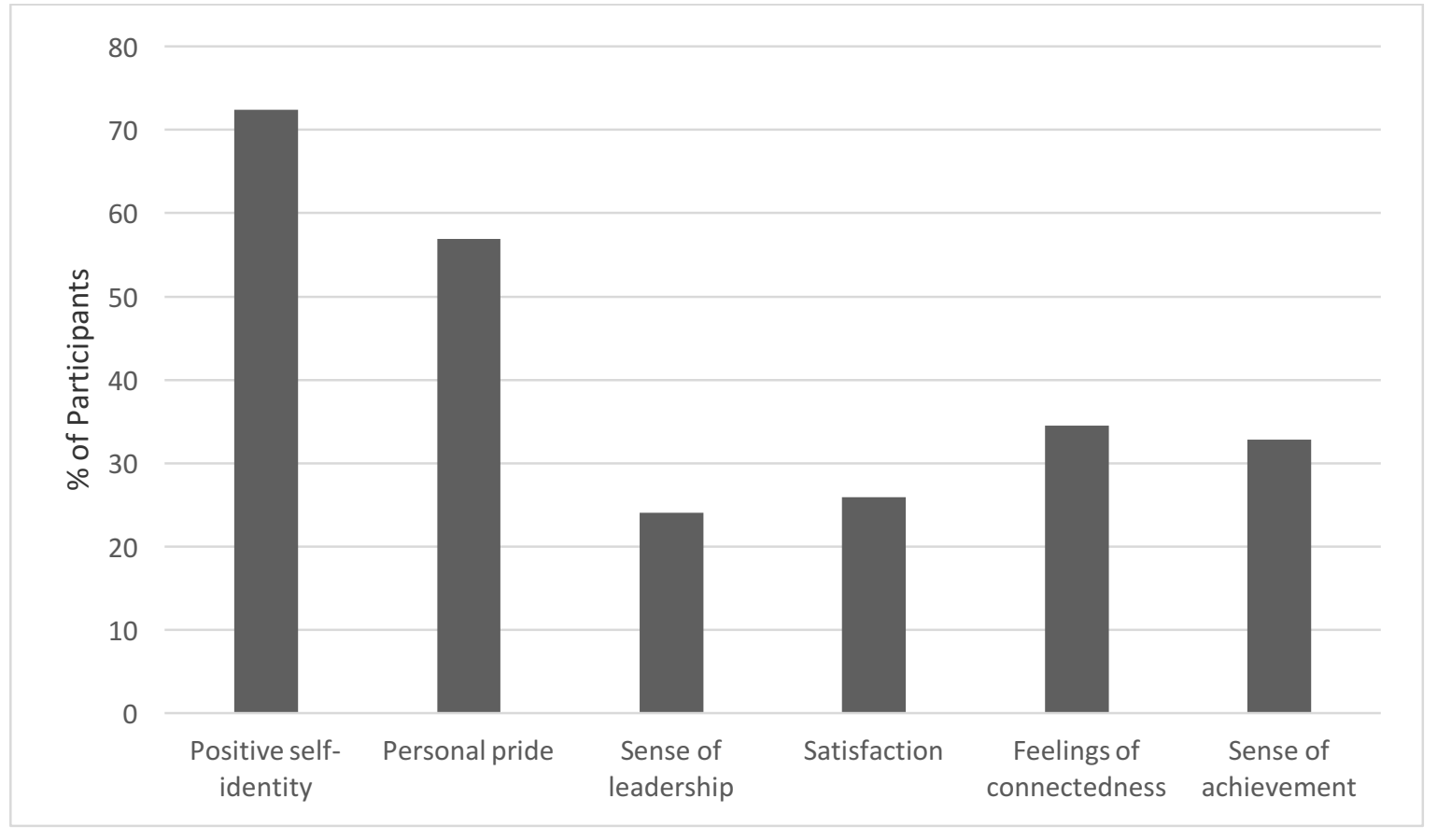

Figure 12: The percentage of participants who identified each anticipated emotion category

\subsubsection{Negative emotions}

As with positive emotions, the coding of anticipated negative emotions if one did not adopt PE behaviour was kept deliberately broad to help inform future research. Negative emotions were themed into six categories (Figure 13): (i) sense of failure linked to feelings of unsuccessfulness, uselessness, feeling unaccomplished, disappointed, dissatisfied and being a 'let down' having "given up" on one's goal: (ii) sense of shame, linked to feeling ashamed, pained and embarrassed: "I'd feel ashamed"; (iii) a sense of sorrow, linked to melancholy and despair: "upset with yourself as you had set it as a goal"; (iv) feelings of guilt by feeling bad or guilty: "feeling a bit like a bad person; guilt that I could have done more"; (v) feeling selfish reflecting on how it would mean they were lazy, careless and ungrateful to others: "selfish and short-sighted; feeling of not joining in with everyone else"; and (vi) feeling annoyed at oneself, linked to frustration and anger: "annoyed at myself; self-directed anger". A seventh category was also identified where participants admitted that they would feel ambivalent if they did not adopt PE behaviours: "unfortunately, I would likely feel indifferent". 


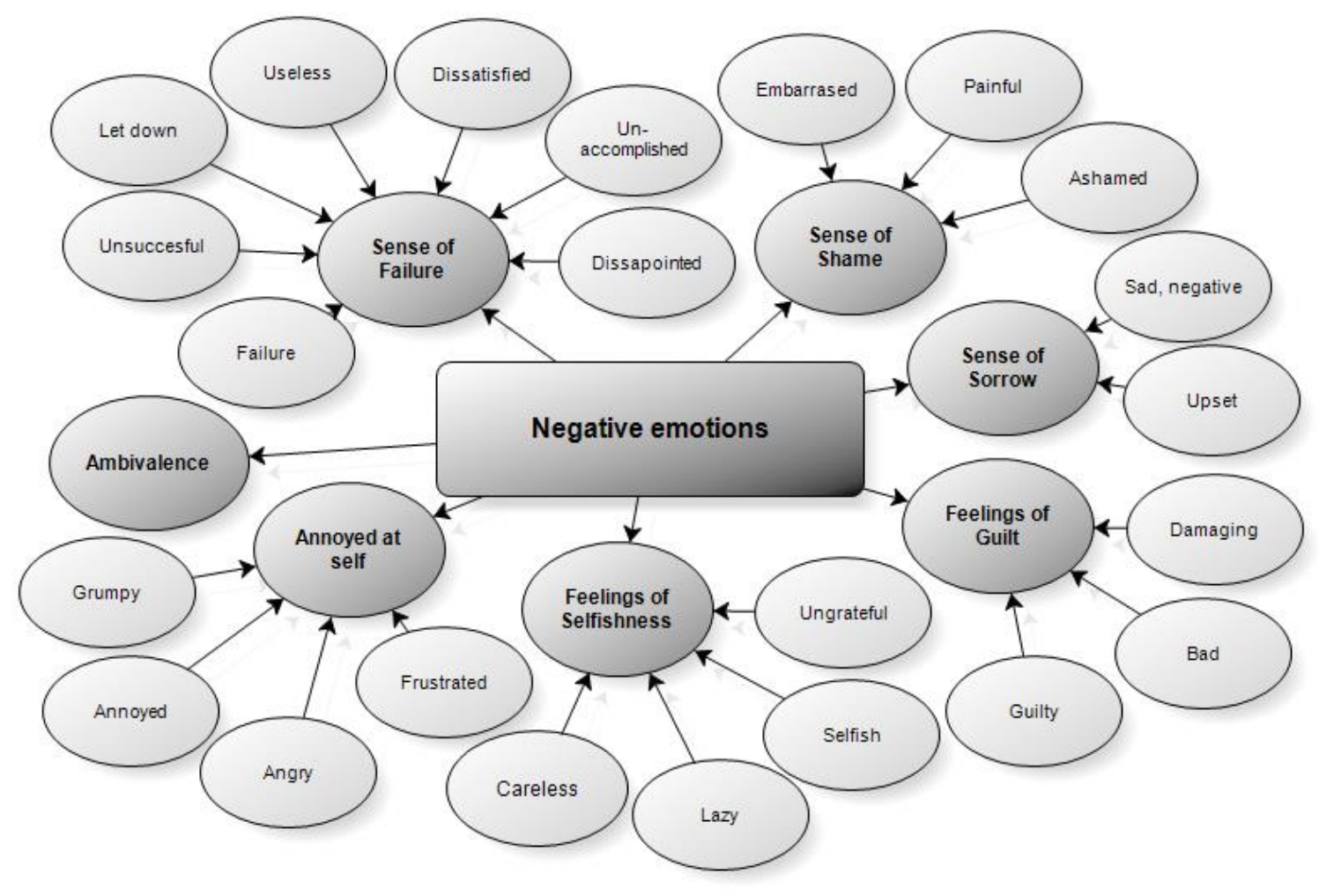

Figure 13: Negative emotions that participants anticipated if they did not adopt PE behaviour

In terms of the prevalence of each category, the most common negative emotion anticipated by participants was a sense of failure (54.7\%) and guilt $(54.7 \%)$, followed by sorrow (24.5\%), being annoyed (24.5\%), shame (17.0\%) and selfishness (11.3\%) (Figure 14).

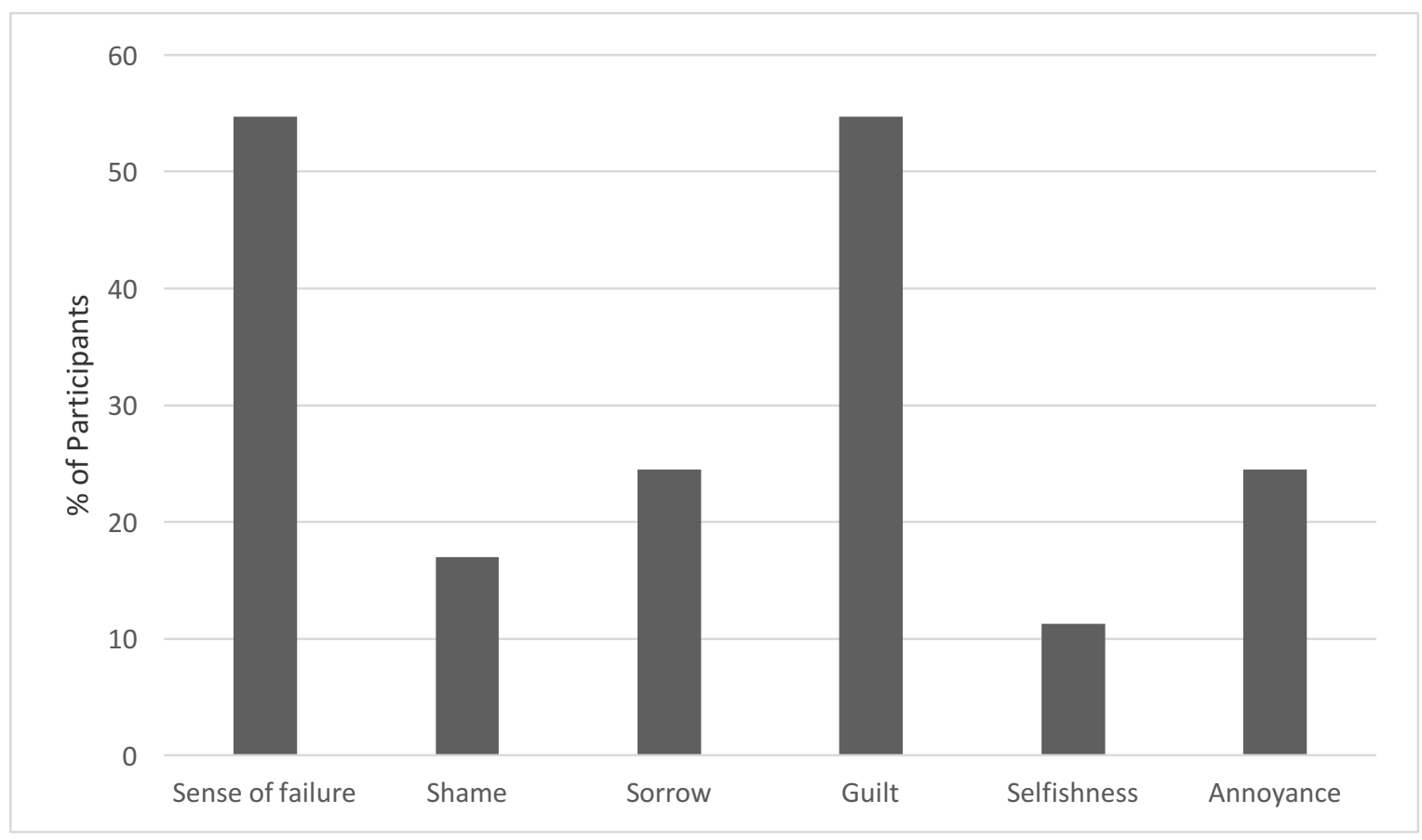


Figure 14: Percentage of participants who identified each negative emotion category

\subsection{Social-emotional referents}

Participants were also asked to think about how they would feel when comparing their own PE behaviour to the behaviour of others, both in terms of if they adopted PE behaviours but others did not and if others' adopted PE behaviour but they did not. It emerged that participants not only anticipated positive feelings at successfully adopting PE behaviour, but also expressed negative emotions towards others if they had not changed their behaviour (Figure 15). Positive emotions included feeling personally successful, happy, smug and superior, pleased, proud and also motivated to help others. Negative emotions included feeling unsupported and helpless, anger, annoyance, sadness, worry, frustration and disappointment directed towards others.

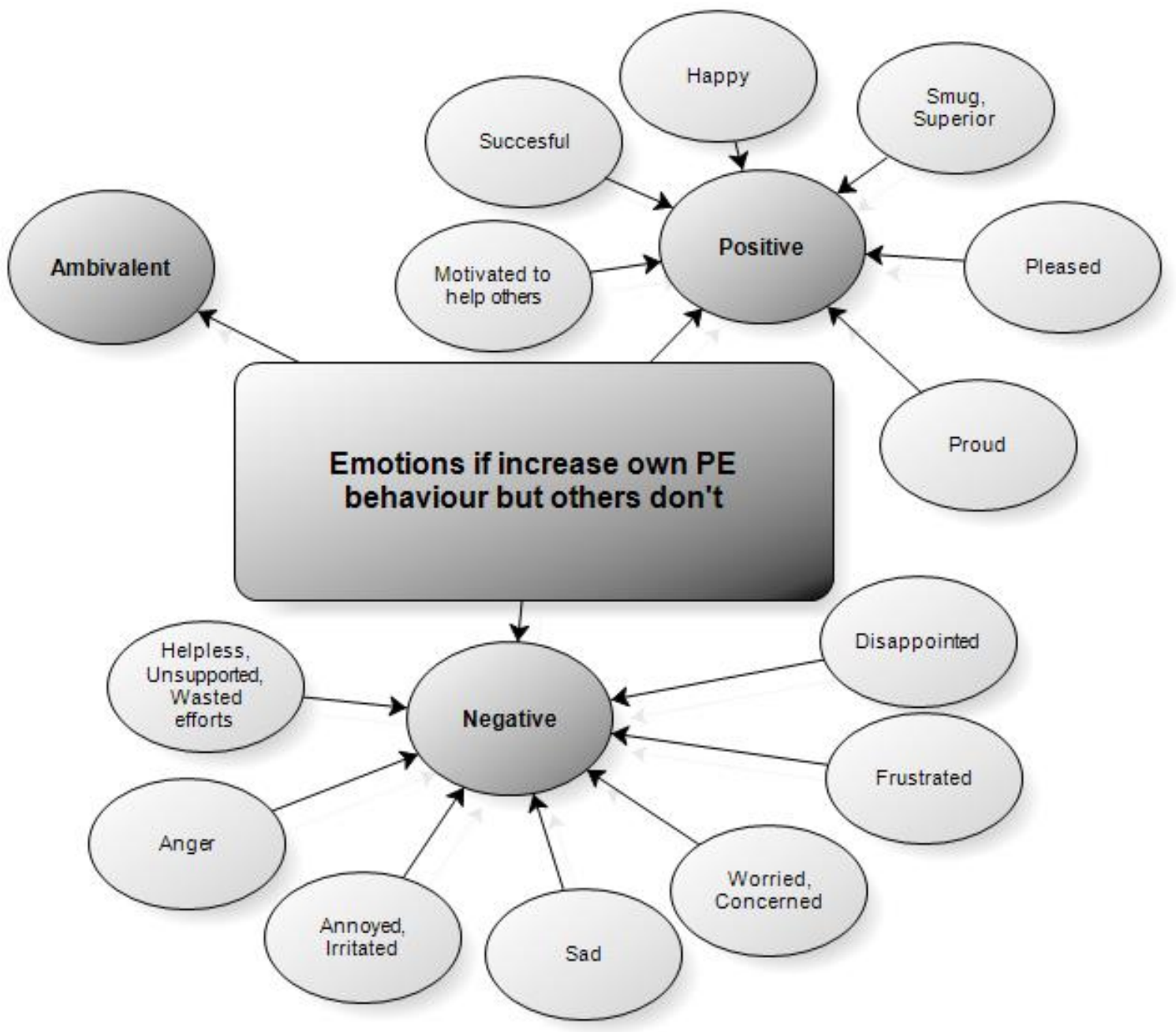

Figure 15: Anticipated emotions if others fail to adopt PE behaviours but participant did 
Contrary to expectations, it emerged that the largest proportion of participants expressed negative emotions when comparing their own PE behaviour to others non-PE behaviour (49.1\%). This compares to $26.3 \%$ who anticipated positive emotions only, $17.5 \%$ who anticipated both positive and negative emotions and 7\% who were ambivalent (Figure 16).

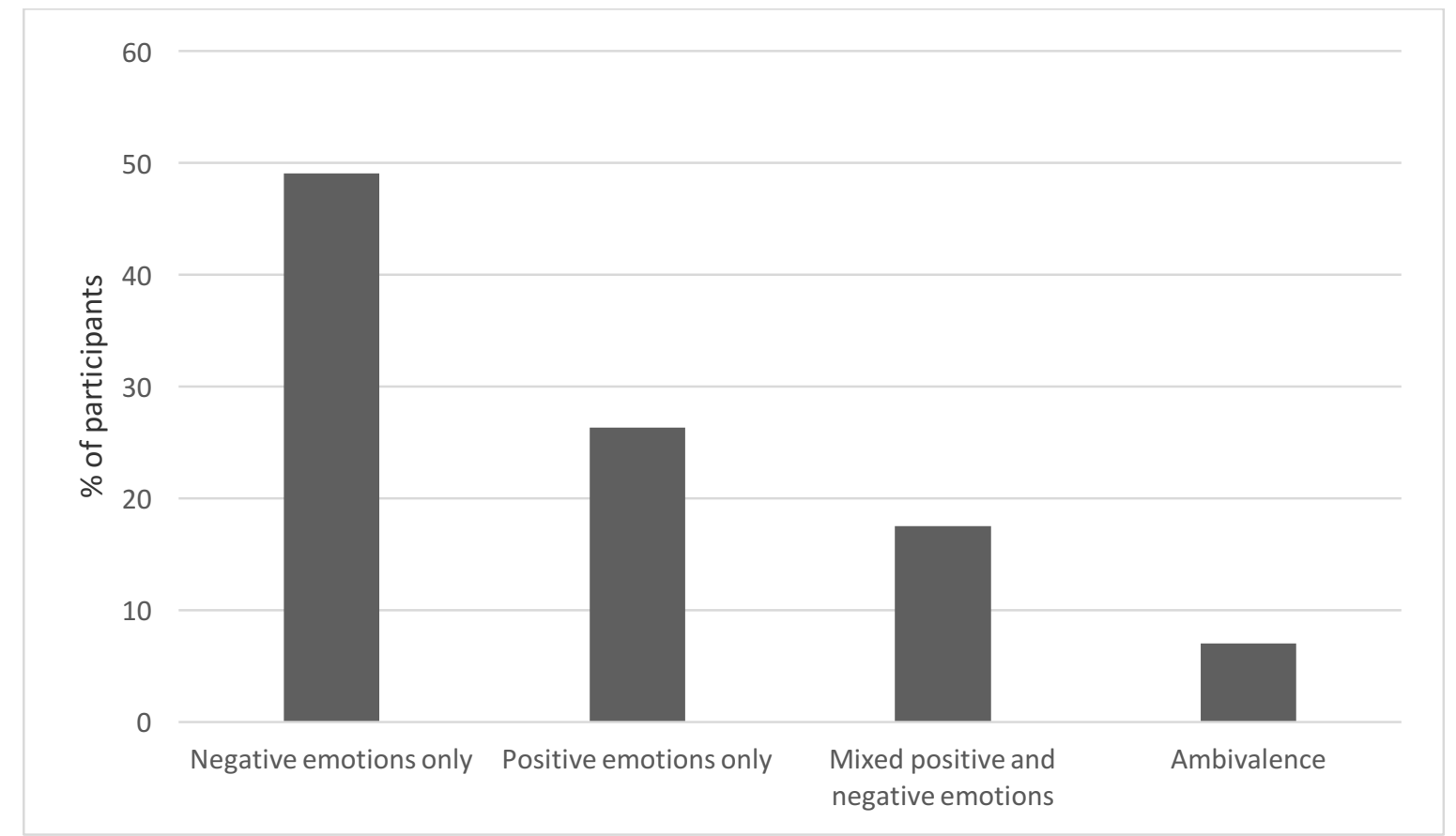

Figure 16: Percentage of participants who anticipated negative emotions only, positive emotions only, mixed emotions or ambivalence

With regards to anticipated emotions that may arise if participants fail to adopt PE behaviours but social others did, again there were both positive and negative anticipated emotions (Figure 17). As expected, negative emotions were directed towards oneself in terms of sadness, annoyance, jealousy, disappointment, guilt, sense of failure, lazy, angry and feeling alone. However, participants also anticipated positive emotions linked to feeling pleased for others, proud of others, relief for reducing their own responsibility to act and inspiration to act like others. 


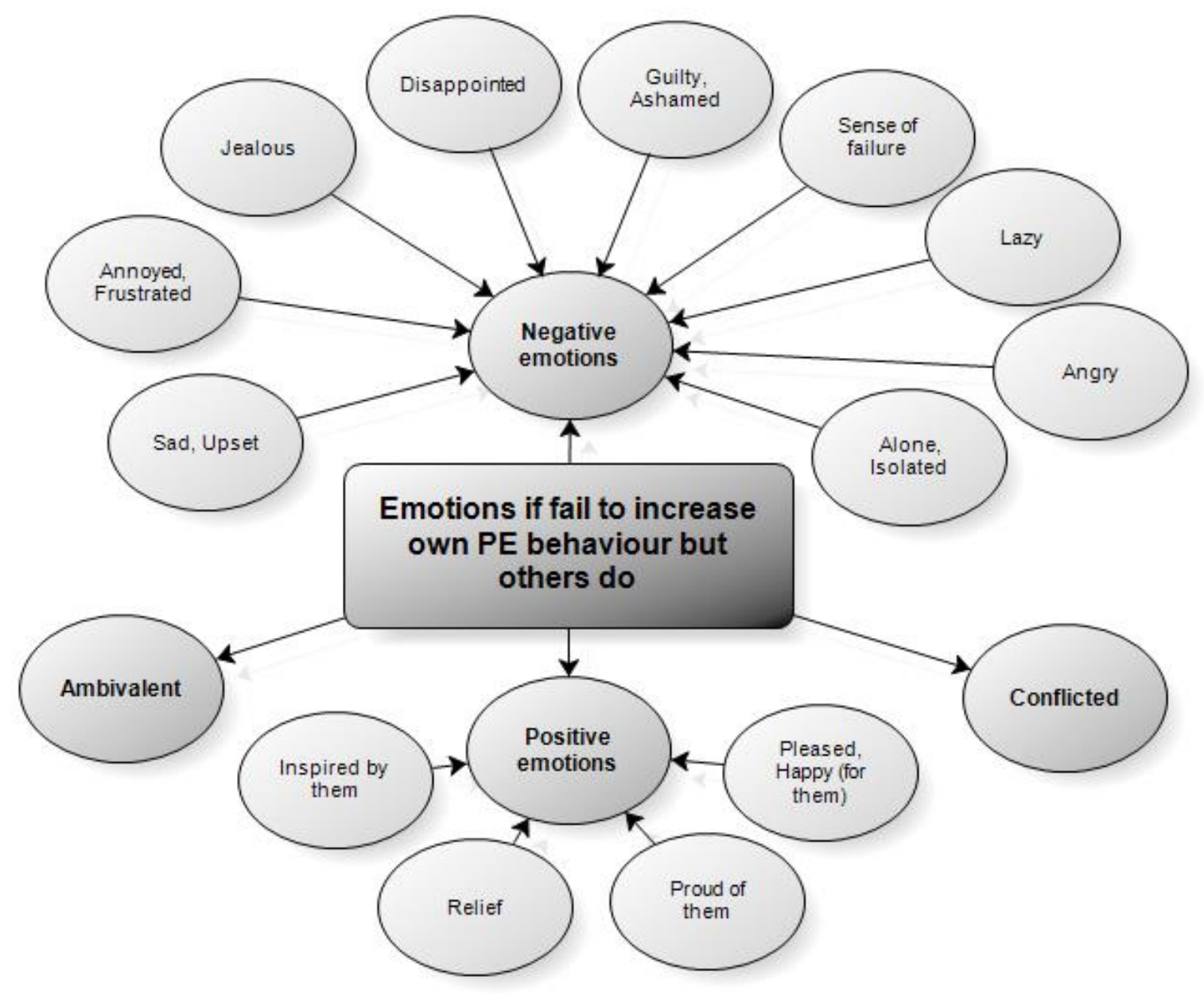

Figure 17: Anticipated emotions if participants failed to change their own behaviour to act in a sustainable way, but others were successful

It emerged that although some participants anticipated only positive or negative emotions, some participants were 'conflicted' in their emotions: "pleased for them, disappointed in myself". The largest proportion of participants expressed negative emotions only (42.1\%), yet unexpectedly $28.1 \%$ expressed positive emotions only and $24.6 \%$ showed conflicted positive and negative emotions (Figure 18). Collectively, this means that over half the sample (52.7\%) identified positive emotions despite their own failed attempts to adopt PE behaviour. This suggests that individuals can think about PE behaviour in a non-competitive and more pro-social way; thus explaining why individuals feel positively as a result of the vicarious success of others. 


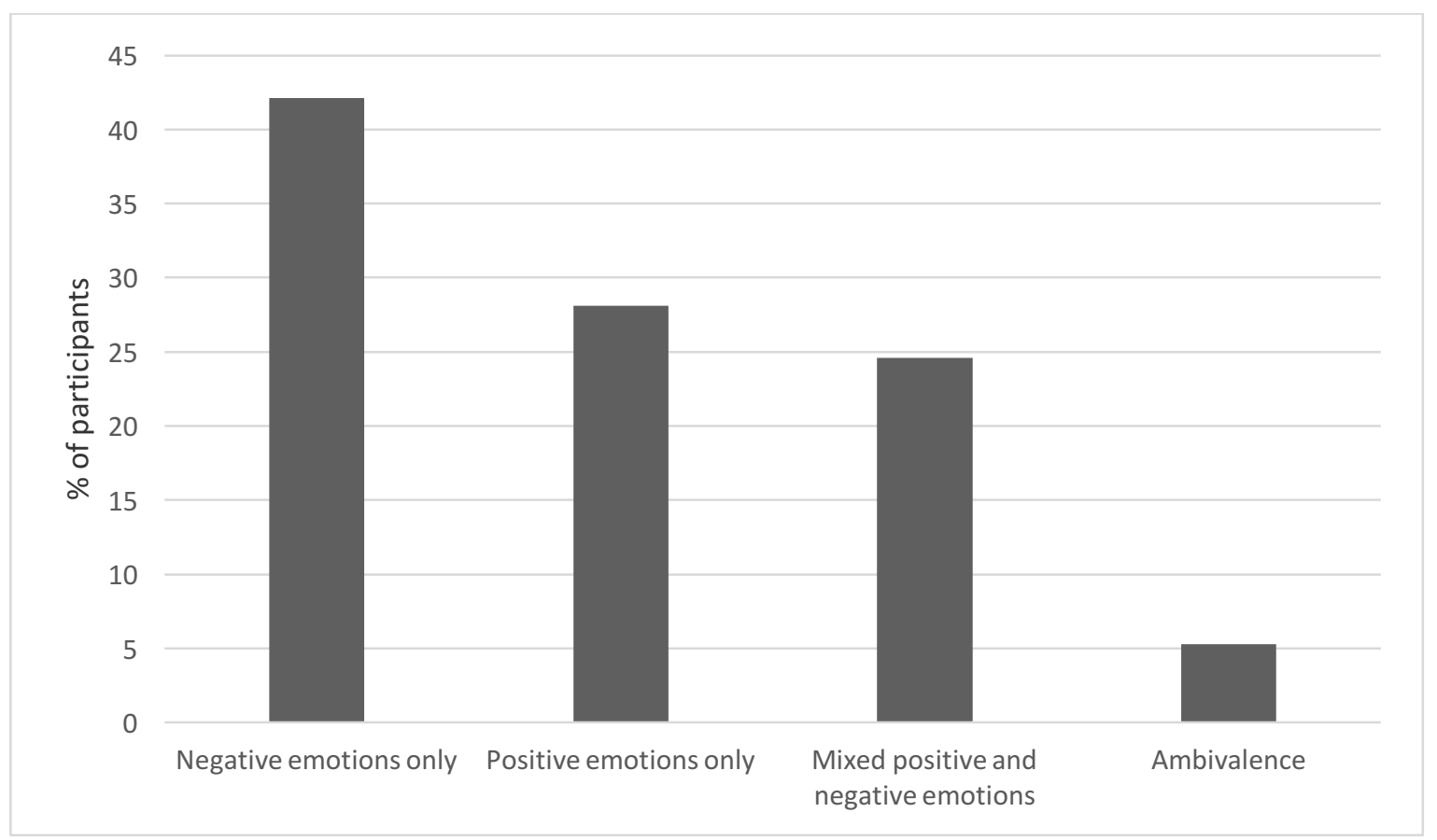

Figure 18: Percentage of participants who anticipated negative emotions only, positive emotions only, conflicted emotions or ambivalence

\section{Discussion}

This study explored in an open-ended way how individuals conceptualise sustainable behaviours. One clear finding from this paper was that participants had significantly higher scores for environmental concern compared to environmental behaviour. This means that participants reported being concerned about the environment, but acknowledged that they did not take active steps to reduce their carbon footprint. Previous research has identified an intention-behaviour gap when considering the adoption of sustainable behaviour (Bamberg 2003). It has been hypothesised in the past that this gap may be due to a dissociation between explicit and implicit attitude - explicit self-report attitudes to the environment may be positive, but underlying implicit attitudes may not, and these implicit attitudes may be more influential for guiding certain aspects of behaviour (Beattie 2010; Beattie and McGuire 2015). However, as participants appeared to report this contradiction by explicitly scoring their environmental behaviour as lower than environmental concern, it could also be explained by a lack of awareness of how to act in a sustainable way. The facts of global warming have been made clear to the public via public dissemination of information through the media and government. Indeed, the recent 'COP21' United Nations Summit in Paris, which set out an international agreement between member states for how to keep global warming below $2^{\circ} \mathrm{C}$, was extensively published on both national and international news channels. The coalition government in the 
U.K. in 2010 further set out to be the 'greenest government ever' through their 'Greening Government Commitments' that sought to cut emissions and make the government more sustainable; albeit the final summary suggested that they failed to hit their targets over the five year period (HM Government 2015). Yet despite government-level commitments to sustainability, the availability of information on how members of public can easily change their behaviour in a more sustainable way is still much less prevalent; indeed this was a theme that was identified by participants when asked about things that might make it easier or enable them to act in a more sustainable way.

Previous research on 'implementation intentions' suggests that intentions are derailed when individuals lack the knowledge on how to adopt intended behaviours (Carrington et al. 2010). Implementation intentions reflect knowledge on 'if/then' rules when making decisions in the real world; a lack of information on how to perform the 'then' behaviour means that intentions are not able to come to fruition (Carrington et al. 2010). Of the types of PE behaviours identified by participants, the two smallest categories of behaviour were purchasing and food-related behaviour, even though these two behaviours are common everyday behaviours that participants readily perform. This seemingly reflects a lack of awareness that one could make behaviour more sustainable by changing everyday shopping and food-related behaviour. A study looking at carbon food labelling in Australia found that householders were generally unaware of the differences between high and low carbon grocery products (see also Beattie 2012b; Beattie, McGuire and Sale 2010). However, a traffic light colour system on product labelling helped them to learn about their shopping behaviour (Sharp and Wheeler 2013). Our study suggested that carbon labelling on grocery products is essential, as individuals are unlikely to consider carbon emissions when buying groceries unaided. As such, it would seem that people lack specific 'implementation intentions' when considering shopping and food related behaviours as ways to improve sustainability. However, carbon labelling clearly does need to be simple (such as the traffic signal approach) to have the desired effect in the time frame required for supermarket shopping. Carbon labelling that is not simple does not seem to attract the visual attention of consumers in that very short time frame, (Beattie 2012b), except for those with the necessary very positive implicit attitude (Beattie and McGuire 2015).

On the other hand, the two largest categories of behaviours identified by participants were 'recycling' and 'travel'; yet, although these behaviours were the most obvious to participants, they were also the ones that face numerous practical barriers that may limit the opportunity for individuals to perform these PE behaviours. For example, a study using the 
TPB to explore commuting behaviour found that people who lived within a two-mile radius of their workplace were significantly more likely to walk to work compared to those who lived within an eight-mile radius (Donald et al. 2014). Indeed when behaviour change is made difficult or inconvenient then it can lead to perceptions that there is 'no point' in trying to change behaviour. A study on bicycle commuting in Ireland found that variability in the cycling to work was better explained by an individual's reasons for not cycling (e.g. inconvenience) rather than reasons to adopt cycling (Claudy and Peterson 2014). It could be suggested that greater public education and information on the various different ways one may adopt PE behaviours would be beneficial by providing the public with information about a greater range of behaviours that they could engage in. This would allow individuals to change their behaviour in ways that are easier for them to adopt and increase collective changes in PE behaviours based on the capacity of the individual.

Interestingly, those who identified purchasing and food-related behaviours had significantly higher scores for environmental concern. This suggests that those who are concerned about the environment seem to have a greater awareness of how to act in a sustainable way, possibly, as they have actively sought information themselves on how they might change their behaviour leading to a greater awareness of different behavioural options. Yet again, there was no relationship between the identification of these PE behaviours and reports of environmental behaviour. Similarity, age was positively correlated with environmental concern but not environmental behaviour; indicating high awareness of the issue of global warming but a failure to adopt sustainable behaviours. Interestingly, this contradicts previous findings, which suggests that age is positively correlated with environmental behaviour (Pinto, Nique, Añaña and Herter 2011) but negatively with environmental concern (Arcury and Christianson 1993). However, this may be due to the characteristics of our particular sample whose mean age was twenty-five years and thus could be considered a 'younger' cohort in the context of research correlating age and environmental concern (Wright, Caserta and Lund 2003).

This study also found that the identification of different types of PE behaviours was positively correlated with both the identification of multiple advantages for adopting PE behaviours and an awareness of multiple disadvantages that may arise from changing behaviour. This suggests an interesting paradox whereby those with greater environmental concern have greater overall knowledge about sustainable behaviours, which may counterintuitively impede behavioural change as they are aware of the potential negative outcomes for 
adopting sustainable behaviours. Indeed, one might suggest that instead of providing individuals with increased information to encourage PE choices, that the design of behavioural 'nudges' by, for example, making meat-free options at a cafeteria the default option, may be more useful for increasing PE behaviours (Campbell-Arvai, Arvai and Kalof 2014). Alternatively, it is possible that our findings may be explained by a methodological issue: environmental behaviour was measured by a single self-report Likert scale item and not measured by asking participants to identify which sustainable behaviours they adopt, it could be that those high on environmental concern judge themselves more harshly in terms of their environmental behaviour. They may objectively perform more sustainable behaviours than those low on environmental concern, but subjectively feel that it is not enough. Further research to unpack the relationship between environmental concern, green awareness and education and $\mathrm{PE}$ behaviour would be useful, especially with regards to designing interventions to encourage behaviour change.

One other finding concerning environmental concern relates to an unusual and nonsignificant trend in the data on participants' 'dwelling location'. Participant means suggested that rural dwellers had the highest scores, followed by suburban dwellers and urban dwellers. This links to previous research on individual differences, which has suggested that those brought up in rural areas score highly for measures on their 'affective connection to nature' (Hinds and Sparks 2008). It suggests that those who are exposed to rural environments develop a greater connection with the environment, which may increase their commitment to protecting nature. Research on visual preference has also identified that rural dwellers, compared to the general population, have a higher preference for 'fractal' shapes, which are linked to complex visual patterns that are associated with 'natural environments' (e.g. tree branches) (Street 2015). Although the trend found in this paper was non-significant, this may be due a sampling issue as the majority of participants identified themselves as 'suburban'. Further research that seeks to compare directly these groups through targeting sampling of rural, suburban and urban dwellers may help to unpack this intriguing relationship. It may be that greater exposure to nature can lead to higher environmental concern and behaviour, and thus the scope for interventions that help to expose individuals to natural environments offers and exciting possibility for encouraging PE behaviour change.

\section{Barriers to PE behaviour change}


Two important questions included in the survey asked participants to identify factors that might make it easier, or more difficult for them, to perform sustainable behaviours. This has implications in terms of understanding how to encourage PE behaviour, by removing some of the possible barriers and enabling pathways to action. Indeed, research suggests that reasons for not adopting sustainable behaviours may have a greater influence on behaviour than reasons for adopting sustainable behaviours (Claudy and Peterson 2014). The largest barrier to PE behaviour identified in this study was related to 'additional costs' linked to both time and money. Similarly, the largest 'enabling' factor that participants identified that might to help them act in a sustainable way was greater access to facilities. Taken together, it seems that participants are willing to adopt PE behaviours, but only if their ability to perform behaviours is made easy and does not impose costs upon them.

An example of how recycling behaviour has been made easier in various countries is by providing 'curb side' recycling facilities to make recycling both easy and time efficient. The Household Waste Recycling Act (2003) in the U.K. ensures that local councils provide curbside recycling facilities to make recycling easier, more accessible and more prevalent in UK households. Interestingly, a report co-led by the National Union of Students in the U.K. found that UK university students were half as likely as the rest of the UK to recycle, with $39 \%$ of students stating the largest barrier was lack of access to easy to use recycling facilities (SITA 2013). This suggests that, although individuals may be concerned about the environment, they lack an emotive desire to make sacrifices in order to achieve green goals. To overcome this, it has been found that activating 'green values' by exposing people to images of nature lowered their price sensitivity to more expensive green electric vehicles (Hahnel, Ortmann, Korcaj and Spada 2014). At a governmental level, it has been found that government taxes can 'nudge' consumer behaviour towards green objectives; for example, the $5 p$ charge on plastic carrier bags that was introduced in England in the UK has had previous success in Wales, which saw a 79\% reduction in plastic bag consumption over 3 years (DEFRA 2015). Further research is required to explore different ways that individuals may be persuaded to adopt more sustainable behaviours via the manipulation of variables related to cost and ease.

The potential benefits to adopting PE behaviour were also themed into two temporal ways of thinking: near-present benefits, which were related to gains that the individual may experience themselves (e.g. cleaner environment); and future-oriented benefits that tended to be more abstract, vague and impersonal (e.g. 'save the planet'). We found that over a third of participants only considered benefits relating to the distant future, which were more abstract 
and vague. Indeed, research has found that individuals tend to think about global warming as a distant problem rather than one that will affect them personally (Hardisty and Weber 2009; Milfont et al. 2012). It could be argued that those who only perceive long-term and distant advantages to changing behaviour will be less likely to act than those who anticipate more concrete and immediate benefits to behaviour change. According to construal level theory (Liberman and Trope 1998), people base their judgements in the world upon their perceived psychological distance to the given stimulus; the further the psychological distance, the more abstract and less connected they feel to the stimulus (Trope and Liberman 2010). Furthermore, when people think about behaviour in the future they tend to favour desirable or ideal goals; whereas proximal behaviours that they are expected to perform now are thought about in terms of how feasible they are (Lutchyn and Yzer 2011). In linking this to the MGB (Perugini and Bagozzi 2001), this suggests that one's self-construal with regards to the perceived psychological distance to the effects of global warming may influence the likelihood that they will perform PE behaviour. Those who perceive it as a psychologically distant problem, with associated benefits to behaviour change not directly affecting them, may desire to act in a sustainable way but lack intentions to perform PE behaviours; whereas those who perceive it as being a proximal problem, with the benefits for behaviour change directly affecting them, may have greater concrete intentions to act. There is further evidence to suggest individual differences in the way people tend to think about the world, with some people focusing on the distant consequences of their behaviour and others focusing on more immediate implications (e.g. Strathman, Fleicher, Boninger and Edwards 1994). The interaction between state and trait temporal perspective, desires and intentions is an interesting avenue for investigation regarding PE choice.

\section{Environmental behaviour as a social construct}

A final implication of this study relates to the way that participants appear to conceptualise PE behaviour as a form of social behaviour, construed in terms of its social dimension. We found that over half of the participants anticipated positive emotions when they failed to adopt PE behaviour but when others succeeded, whereas participants often anticipated negative emotions when they succeeded in adopting PE behaviour but when others failed. We also found when participants were asked about their own PE behaviour only (i.e. with no comparison to others), anticipated positive outcomes not only related to their personal wellbeing (e.g. pride, happiness) but were also linked to their social identity - they felt more 'connected' to others and more like a 'leader'. It could be suggested that sustainable behaviours 
are often perceived as essentially social rather than an individual form of behaviours and so focus on the performance of the group rather than on individual behaviour might be very important.

It has previously been reported that when one perceives one's social group as being responsible for protecting the environment then this increases feelings of pride and greater ingroup favouritism; whereas if one feels their peers are responsible for harming the environment it can lead to feelings of guilt and anger (Harth, Leach and Kessler 2013). The findings from this study extend these results, suggesting that the groups' environmental behaviour has a greater effect on emotions than individual behaviour, meaning that one's emotions align with the performance of the group rather than being just a reflection of personal performance. With regards to differences between individualist and collectivist cultures, individuals from collectivist cultures hold greater intentions to perform PE behaviours if they perceive it to be a social norm, compared to individualist cultures that were more influenced by attitude formations (Mancha and Yoda 2015). It has been suggested in the past that interventions should be designed in line with these observations by placing greater emphasis on the social benefits in collectivist cultures and attitude formation in individualist cultures. Interestingly, data from our study indicated that participants considered PE behaviour as a collective and social behaviour, despite being from the UK (an individualist culture). It is possible that because global warming, by definition, affects everyone, that even those from traditionally individualist cultures perceive it as a form of social behaviour affecting the global community and so conceptualise it within a social lens.

We would, therefore, suggest that interventions to increase PE behaviour should focus on large-scale and collective efforts that communities as a whole could engage with (e.g. community recycling facilities, reduced packaging on products in supermarkets) rather than individual behaviours (e.g. cycling to work, turning out lights). Indeed, 22.8\% of participants identified feelings of 'helplessness' if others failed to adopt PE behaviours, which may explain why people fail to adopt individual PE behaviours if they feel they are pointless in comparison to the behaviour of the group. Thus, rather than interventions to try to increase individual PE behaviours (e.g. switch off your lights), there needs to be a greater focus on the fostering of green communities, be that led from the government, large organisations (e.g. supermarkets) or workplace schemes. Furthermore, given that the anticipation of positive emotions has a greater influence on behaviour than negative emotions (Koenig-Lewis et al. 2014), interventions could well focus on positive emotional outcomes that will arise from collective 
PE behaviour. Rather than making individuals fearful of climate change, or focus on the anticipation of guilt for not adopting PE behaviours, interventions should aim to highlight the emotional benefits one may experience as a result of adopting behaviour related to a sense of communal good. This could foster feelings of social cohesion and sustainable cultural norms, thus reducing negative emotions associated with others' non-PE behaviours and increasing positive feelings of collective efforts.

\section{Conclusion}

This paper has provided a detailed, qualitative exploration of how individuals conceptualise behaviours related to environmental behaviour, sustainability and global warming. Previous studies in psychology have mainly focussed on testing theoretical models of planned behaviour, which means their methodologies essentially 'prime' participants to think about certain types of behaviour (e.g. recycling), and in the real-world a gap between intentions to adopt PE behaviours and the actual uptake of behaviours repeatedly appears. We argue that this may be due to the lack of salience given to these behaviours when individuals make decisions in their day-to-day lives. The present study, therefore, offers a unique perspective on the 'free' responses of participants when asked to think about various aspects of PE behaviour. It suggests amongst other things that sustainable behaviour is conceptualised by individuals as a form of 'social behaviour' and this finding alone may well have significant implications for the design of interventions to increase sustainable behaviours amongst the public. As we said in the introduction, the search for the means to limit climate change must be a number one global priority, and engaging the public in this to the degree that it leads to large-scale behaviour change on their part is of central importance. This paper offers some new and original considerations in this regard. 


\section{References}

Ajzen, Icek. 1991. The theory of planned behaviour. Organizational Behavior and Human Decision Processes 50. 179-211.

Ajzen, Icek. 2002. Constructing a theory of planned behaviour questionnaire. http://people.umass.edu/aizen/pdf/tpb.measurement.pdf Accessed on $19^{\text {th }}$ October 2015.

Allport, Gordon. W. 1935. Attitudes. In C. Murchison (Eds.), Handbook of Social Psychology. Massachusetts: Clark University Press.

Arcury, Thomas. A. \& Eric H. Christianson. 1993. Rural-urban differences in environmental knowledge and actions. The Journal of Environmental Education 25. 19-25.

Armitage, Christopher. J \& Mark Conner. 2001. Efficacy of the theory of planned behaviour: a meta-analytic review. British Journal of Social Psychology 40. 471-499.

Arnocky, Steven, Taciano L. Milfont \& Jeffrey R. Nicol. 2014. Time perspective and sustainable behaviour: Evidence for the distinction between consideration of immediate and future consequences. Environment and Behavior 46. 556-582.

Auger, Pat \& Timothy M. Devinney. 2007. Do what consumers say matter? The misalignment of preferences with unconstrained ethical intentions. Journal of Business Ethics 76. 361-383.

Bamberg, Sebastian. 2003. How does environmental concern influence specific environmentally related behaviors? A new answer to an old question. Journal of Environmental Psychology 23. 21-32.

Barthes, Roland. 1957. Mythologies. Trans. Annette Lavers. New York: Hill.

Beattie, Geoffrey. 2010. Why Aren't We Saving the Planet? A Psychologist's Perspective. London: Routledge.

Beattie, Geoffrey. 2011. Making an action film: Do films such as Al Gore's An Inconvenient Truth really make any difference to how we think and feel about climate change? Nature Climate Change 1. 372-374. 
Beattie, Geoffrey. 2012a. Our Racist Heart? An Exploration of Unconscious Prejudice in Everyday life. London: Routledge.

Beattie, Geoffrey. 2012b. How effective is carbon labelling for the consumer? Nature Climate Change 2. 214-217.

Beattie, Geoffrey., Doron Cohen \& Laura McGuire. 2013. An exploration of possible unconscious ethnic biases in higher education: The role of implicit attitudes on selection for university posts. Semiotica $197.171-201$.

Beattie, Geoffrey \& Laura McGuire. 2012. See no evil? Only implicit attitudes predict unconscious eye movements towards images of climate change. Semiotica 192.315339.

Beattie, Geoffrey \& Laura McGuire. 2015. Harnessing the unconscious mind of the consumer: How implicit attitudes predict pre-conscious visual attention to carbon footprint information on products. Semiotica 204, 253-290.

Beattie, Geoffrey., Laura McGuire \& Laura Sale. 2010. Do we actually look at the carbon footprint of a product in the initial few seconds? An experimental analysis of unconscious eye movements. International Journal of Environmental Cultural Economic and Social Sustainability 6. 47-66.

Beattie, Geoffrey \& Laura Sale. 2009. Explicit and implicit attitudes to low and high carbon footprint products. International Journal of Environmental Cultural Economic and Social Sustainability 5. 191-206.

Beattie, Geoffrey \& Laura Sale. 2011. Shopping to save the planet? Implicit rather than explicit attitudes predict low carbon footprint consumer choice. International Journal of Environmental Cultural Economic and Social Sustainability 7. 211-232.

Beattie, Geoffrey., Laura Sale \& Laura McGuire. 2011. An inconvenient truth? Can extracts of a film really affect our psychological mood and our motivation to act against climate change? Semiotica 187 . 105-125.

Braun, Virginia \& Victiria Clarke. 2006. Using thematic analysis in psychology. Qualitative Research in Psychology 3. 77-101. 
Campbell-Arvai, Victoria., Joseph Arvai \& Linda Kalof. 2014. Motivating Sustainable Food Choices: The Role of Nudges, Value Orientation, and Information Provision. Environment and Behavior 46. 453-475.

Carrington, Michal J. Benjamin A. Neville \& Gregory J. Whitwell. 2010. Why ethical consumers don't walk their talk: Towards a framework for understanding the gap between the ethical purchase intentions and actual buying behaviour of ethically minded consumers. Journal of Business Ethics 97. 139-158.

Carrus, Giuseppe, Paola Passafaro \& Mirilia Bonnes. 2008. Emotions, habits and rational choices in ecological behaviours: The case of recycling and use of public transportation. Journal of Environmental Psychology 28. 51-62.

Cheng, Mei-Fang \& Pei-Ju Tung. 2010. The moderating effect of perceived lack of facilities on consumers' recycling intentions. Environment and Behavior 42. 824-844.

Claudy, Marius. C. \& Mark Peterson. 2014. Understanding the Underutilization of Urban Bicycle Commuting: A Behavioral Reasoning Perspective. Journal of Public Policy \& Marketing 33. 173-187.

Department for Environment Food and Rural Affairs. 2015.

https://www.gov.uk/government/publications/single-use-plastic-carrier-bags-whywere-introducing-the-charge/carrier-bags-why-theres-a-5p-charge. Accessed $26^{\text {th }}$ January 2016

Donald, Ian J., Simon R. Cooper \& Stacey Conchie. 2014. An extended theory of planned behaviour model of the psychological factors affecting commuters' transport mode use. Journal of Environmental Psychology 40. 39-48.

Elgaaied, Leila. 2012. Exploring the role of anticipated guilt on pro-environmental behaviour - a suggested typology of residents in France based on their recycling patterns. Journal of Consumer Marketing 29. 369-377.

Elliot, Andrew. 2006. The hierarchical model of approach-avoidance motivation. Motivations and Emotion 30. 111-116. 
Fazio, Russell. H. 1990. Multiple processes by which attitudes guide behavior: The MODE model as an integrative framework. Advances in Experimental Social Psychology 23. 75-109.

Fransson, Niklas., Per Davidsson., Agneta Marell \& Tommy Garling. 1994. Environmental concern: Conceptual definition, measurement methods and research findings. Goteborg: Department of Psychology, University of Goteborg.

Greenwald, Anthony., Debbie E. McGhee \& Jordan L. Schwartz, J. L. 1998. Measuring individual differences in implicit cognition: The implicit association test. Journal of Personality and Social Psychology 74. 1464-1480.

Greenwald, Anthony. G., Andrew T. Poehlman, Eric L. Uhlmann \& Mahzarin R. Banaji. 2009. Understanding and using the Implicit Association Test: III. Meta-analysis of predictive validity. Journal of Personality and Social Psychology 1. 17 - 41.

Hahnel, Ulf. J., Céline Ortmann, Liridon Korcaj \& Hans Spada. 2014. What is green worth to you? Activating environmental values lowers price sensitivity towards electric vehicles. Journal of Environmental Psychology 40. 306-319.

Hardisty, David .J. \& Elke. U. Weber. 2009. Discounting future green: Money versus the environment. Journal of Experimental Psychology: General 138. 329-340.

Harth, Nicole .S., Colin. W. Leach. \& Thomas Kessler. 2013. Guilt, anger, and pride about in-group environmental behaviour: Different emotions predict distinct intentions. Journal of Environmental Psychology 34. 18-26.

Hegarty, John. 2011. Hegarty on Advertising: Turning Intelligence into Magic. London: Thames and Hudson

Hinds, Joe \& Paul Sparks. 2008. Engaging with the natural environment: The role of affective connection and identity. Journal of Environmental Psychology 28. 109-120.

IPCC (2015). Climate Change 2014: Synthesis Report. Contribution of Working Groups I, II and III to the Fifth Assessment Report of the Intergovernmental Panel on Climate Change IPCC: Geneva, Switzerland.

Joireman, Jeff., Daniel Balliet, David Sprott, Eric Spangenberg \& Jenifer Schultz. 2008. Consideration of future consequences, ego-depletion, and self-control: Support for 
distinguishing between CFC-Immediate and CFC-Future sub-scales. Personality and Individual Differences 45. 15-21.

Kaiser, Florian.G., Wesley Schultz, Jaime Berenguer, Victor Corral-Verdugo., \& Geetika Tankha 2008. Extending planned environmentalism. Anticipated guilt and embarrassment across cultures. European Psychologist 13. 288-297.

Koenig-Lewis, Nicole., Adrian Palmer, Janine Dermody \& Andreas Urbye. 2014. Consumers' evaluations of ecological packaging - Rational and emotional approaches. Journal of Environmental Psychology 37. 94-105.

Lewin, Kurt. 1939. Field theory and experiment in social psychology: concepts and methods. American Journal of Sociology 44. 868-896.

Liberman, Nira, \& Yaacov Trope. 1998. The role of feasibility and desirability considerations in near and distant future decisions: A test of temporal construal theory. Journal of Personality and Social Psychology 75. 5-18.

Liberman, Nira., Yaacov Trope, Sean Macrae \& Steven Sherman. 2007. The effect of level of construal on temporal distance. Journal of Experimental Social Psychology 43. 143149.

Lutchyn, Yuliya \& Marco Yzer. 2011. Construal level theory and theory of planned behaviour: Time frame effects on salient belief generation. Journal of Health Communication 16. 595-606.

Mancha, Ruben M. \& Carol Y. Yoder. 2015. Cultural antecedents of green behavioural intent: An environmental theory of planned behaviour. Journal of Environmental Psychology 43. 145-154.

Milfont, Taciano .L. \& Valdiney V. Gouveia. 2006. Time perspective and values: An exploratory study of the relations to environmental attitudes. Journal of Environmental Psychology 26. 72-82.

Milfont, Taciano .L., Jessie Wilson \& Pollyane Diniz. 2012. Time perspective and environmental engagement: A meta-analysis. International Journal of Psychology 47. 325-334. 
Oatley, Keith., Dacher Keltner \& Jennifer M. Jenkins. 2006. Understanding emotions $\left(2^{\text {nd }}\right.$ ed.). Blackwell: John Wiley \& Sons.

Peattie, Ken. 2001. Golden goose or wild goose? The hunt for the green consumer. Business Strategy and the Environment 10. 187-199.

Perugini, Marco \& Richard P. Bagozzi. 2001. The role of desires and anticipated emotions in goal-directed behaviours: Broadening and deepening the theory of planned behaviour. British Journal of Social Psychology 40. 79-98.

Perugini, Marco \& Richard P. Bagozzi. 2004. The distinction between desires and intentions. European Journal of Social Psychology 34. 69-84.

Petrocelli, John .V. 2003. Factor validation of the consideration of future consequences scale: Evidence for a short version. Journal of Social Psychology 143. 405-413.

Pinto, Diego. C., Walter M. Nique, Edar da Silva Añaña \& Márcia M. Herter. 2011. Green consumer values: how do personal values influence environmentally responsible water consumption?. International Journal of Consumer Studies 35. 122-131.

Polonsky, Michael .J., Andrea Vocino, Martin Grimmer \& Morgan P. Miles. 2014. The interrelationship between temporal and environmental orientation and proenvironmental consumer behaviour. International Journal of Consumer Studies 38. 612-619.

Pooley, Julie .A. \& Moira O'Connor. 2000. Environmental education and attitudes. Environment and Behavior 32. 711-723.

Richard, René., Joop van der Pligt \& Nanne de Vries. 1996. Anticipated affect and behavioural choice. Basic and Applied Social Psychology 18. 111-129.

Ryan, Gery. W., \& Russel H. Bernard. 2000. Data management and analysis methods. In Norman Densin and Yvonna Lincoln, Eds. Handbook of Qualitative Research, 2nd Ed. / Thousand Oaks, CA: Sage Publications.

Sharp, Anne \& Meagan Wheeler. 2013. Reducing householders' grocery carbon emissions: Carbon literacy and carbon label preferences. Australasian Marketing Journal 21. 240-249. 
SITA. 2013. Sustainability Report. http://www.sita.co.uk/downloads/SITAUKSustainabilityReport2013-1408-web.pdf. Accessed 26 ${ }^{\text {th }}$ January 2016

Stocker, Thomas. F., Dahe Qin, Gian-Kasper Plattner, Melinda Tignor, Simon K. Allen, Judith Boschung \& Pauline. M. Midgley. 2014. IPCC, 2014: Climate Change 2014: The Physical Science Basis. Contribution of Working Group I to the Fifth Assessment Report of the Intergovernmental Panel on Climate Change.

Szmigin, Isabelle., Marylyn Carrigan \& Morven G. McEachern. 2009. The conscious consumer: taking a flexible approach to ethical behaviour. International Journal of Consumer Studies 33. 224-231.

Strathman, Alan., Faith Fleicher, David Boninger \& Scott Edwards. 1994. The consideration of future consequences: weighting immediate and distant outcomes of behaviour. Journal of Personal and Social Psychology 66. 742-752.

Street, Nicola. 2015. What have Fractals got to do with it? Individual Differences in Aesthetic experience. Liverpool, UK: University of Liverpool PhD Thesis.

Trope, Yaacov \& Nira Liberman. 2003. Temporal construal. Psychological Review 110. 403421.

Trope, Yaacov \& Nira Liberman. 2010. Construal-level theory of psychological distance. Psychological Review 117. 440-463.

Tversky, Amos \& Daniel Kahneman. 1974. Judgment under uncertainty: Heuristics and biases. Science 185. 1124-1131.

Unilever. 2013. Unilever Sustainability Plan. http://www.unilever.com.tw/Images/slp Unilever-Sustainable-Living-Plan2013 tcm13-388693 tcm206-395956.pdf accessed $26^{\text {th }}$ January 2016

Usunier, Jean Claude \& Pierre Valette-Florence. 2007. The temporal styles scale: a review of developments and replications over 15 years. Time and Society 16. 333-366.

Walker, Gabrielle \& David King. 2008. The hot topic: How to tackle global warming and still keep the lights on. London: Bloomsbury. 
Wall, Rob., Patrick Devine-Wright \& Greig A. Mill. 2007. Comparing and combining theories to explain pro-environmental intentions: The case of commuting-mode choice. Environment and Behavior 39. 731-753.

Wright, Scott. D., Michael Caserta \& Dale A. Lund, D. 2003. Older Adults' Attitudes, Concerns, and Support for Environmental Issues in the "New West". The International Journal of Aging and Human Development 57. 151-179.

Zimbardo, Philip .G. \& John N. Boyd. 1999. Putting time in perspective: A valid, reliable individual-difference metric. Journal of Personality and Social Psychology 77. 12711288. 